\title{
Towards general scalar-Yukawa renormalisation group equations at three-loop order
}

\section{Tom Steudtner}

Fakultät für Physik, TU Dortmund, Otto-Hahn-Str. 4, D-44221 Dortmund, Germany

Department of Physics and Astronomy, University of Sussex, Pevensey 2, Brighton, BN19QH, U.K.

E-mail: tom2.steudtner@tu-dortmund.de

ABSTRACT: For arbitrary four-dimensional quantum field theories with scalars and fermions, renormalisation group equations in the $\overline{\mathrm{MS}}$ scheme are investigated at threeloop order in perturbation theory. Collecting literature results, general expressions are obtained for field anomalous dimensions, Yukawa interactions, as well as fermion masses. The renormalisation group evolution of scalar quartic, cubic and mass terms is determined up to a few unknown coefficients. The combined results are applied to compute the renormalisation group evolution of the gaugeless Litim-Sannino model.

KeYworDS: Renormalization Group, Renormalization Regularization and Renormalons

ARXiv EPrint: 2101.05823 


\section{Contents}

1 Introduction 1

2 Setup 2

3 Handling of $\gamma_{5} \quad 4$

4 Scalar anomalous dimension $\quad 4$

$\begin{array}{lll}5 & \text { Fermion anomalous dimension } & 6\end{array}$

$\begin{array}{lll}6 & \text { Yukawa interaction and fermion masses } & 7\end{array}$

$\begin{array}{lll}7 & \text { Scalar quartic interactions } & 9\end{array}$

8 Example: Litim-Sannino model $\quad 15$

9 Discussion and outlook $\quad 16$

$\begin{array}{ll}\text { A Conversion to Weyl consistency condition basis } & 17\end{array}$

$\begin{array}{ll}\text { B Comparison to supersymmetric RGEs } & 18\end{array}$

\section{Introduction}

The determination of renormalisation group equations (RGEs) has remained an active field, driven by applications in precision calculations, critical phenomena, model building, grand unification, UV completion and many more. While a plethora of perturbative results exist for theories of special interest, there is also a solid underpinning of two-loop RGEs for all renormalisable quantum field theories (QFTs) [1-8], as well as three-loop order expressions for the gauge sector [9-12]. This is possible due to considering a template action [1-3, 8, 12] of generalised couplings and fields, in which any renormalisable QFT can be embedded. For such a model, loop integrations and spinor summations can be performed explicitly when computing RGEs, while retaining contractions of the generalised couplings. Thus, results are universally reusable with the reduced complication of inserting embedded couplings into template expressions. In fact, this step can be automatised by existing software tools, such as [13-16]. An extension of template framework to the next loop order represents a monumental step which would benefit for all research areas making use of QFT. However, due to the maximal generality of the template action, the computations required involve a high number of Feynman diagrams, which are by themselves technically challenging at three-loop level. Moreover, additional complications due to the $\gamma_{5}$ problem arise [17]. It is 
hence appealing to cut corners by computing RGEs directly for QFTs of interest instead, which however fails to deliver a contribution to the bigger picture.

And yet, such efforts are not in vain. An approach alternative to computing RGEs in the template QFT directly is to formulate a general ansatz for them instead, consisting of all possible combinations of coupling contractions with unknown coefficients. One thus obtains $\beta$ - and $\gamma$-functions depending on these open parameters, which can be compared term by term to existing literature results. The advantage is that such results do not need to be as general as the template theory itself. In fact, input models can be relatively simple, as long as each of them contains enough distinctive features and they are available in sufficient numbers. Hence, the problem of conducting a massive and complicated calculation is broken down into several smaller and independent steps, from which a general result can be collected.

The approach has previously been employed in, e.g., $[12,18,19]$ to study Weyl consistency conditions [20,21], as well as in [22, 23] to compute pure scalar RGEs, and [21] to gain three-loop Yukawa $\beta$-functions for complex scalars and chiral fermions. The former is paving the way to obtain general 4-loop gauge $\beta$-functions - with SM expressions already available [24] — and eventually general 3-loop Yukawa $\beta$-functions. However, three-loop RGEs for the scalar potential are not covered in these efforts. On the other hand, the scalar sector is often not technically natural due to the lack of a protective symmetry, and hence cannot be neglected in RG studies. Therefore, we target scalar quartic and cubic couplings as well as mass terms in this work. Following up on the pure scalar results [22, 23, 25, 26], we also include fermionic contributions through Yukawa interactions and masses, while leaving the entirety of the gauge sector for future studies. As our input, we utilise three-loop results in the Two-Higgs-Doublet Model (THDM) [27], the Standard Model (SM) [28-32], Gross-Neveu-Yukawa theories [33, 34] as well as supersymmetric RGEs [35, 36].

This paper is organised as follows: sections 2 and 3 introduce our notations and conventions. In section 4-7, renormalisation group equations the general scalar-Yukawa models are obtained. As an application, three-loop RGEs for the Litim-Sannino model in the gaugeless limit are computed for the first time in section 8 , before concluding with section 9 .

\section{Setup}

In this work, we consider a general QFT consisting of Weyl fermions $\psi_{i}$ and real scalar fields $\phi_{a}$ with the Lagrange density

$$
\begin{aligned}
\mathcal{L}= & \frac{1}{2} \partial^{\mu} \phi_{a} \partial_{\mu} \phi_{a}+\frac{i}{2} \psi^{j} \tilde{\sigma}^{\mu} \partial_{\mu} \psi_{j}-\frac{1}{2} y^{a j k} \phi_{a}\left(\psi_{j} \varepsilon \psi_{k}\right)-\frac{1}{24} \lambda_{a b c d} \phi_{a} \phi_{b} \phi_{c} \phi_{d} \\
& -\frac{1}{2} \mathrm{~m}^{j k}\left(\psi_{j} \varepsilon \psi_{k}\right)-\frac{1}{2} m_{a b}^{2} \phi_{a} \phi_{b}-\frac{1}{6} h_{a b c} \phi_{a} \phi_{b} \phi_{c},
\end{aligned}
$$

featuring Yukawa couplings $y^{a i j}$, scalar quartic and cubic interactions $\lambda_{a b c d}, h_{a b c}$ as well as fermion and scalar masses $\mathrm{m}^{i j}$ and $m_{a b}^{2}$. Here, $\varepsilon$ denotes the two-dimensional LeviCivita symbol contracting spinor indices, which are not shown explicitly. Each of these interactions are symmetric under permutation of their fermionic $(i, j, k, l, \ldots)$ and scalar indices $(a, b, c, d, \ldots)$, respectively. Moreover, fermion indices in this convention run over 
Weyl components as well as their conjugates. This accounts for the additional factor $\frac{1}{2}$ and $\tilde{\sigma}^{\mu}=\sigma^{\mu}$ or $\bar{\sigma}^{\mu}$ in the kinetic term, as well the missing complex conjugation of the fermion mass and Yukawa interactions. Fields and couplings

$$
\psi^{i}=\psi_{i}^{*}, \quad y^{a j k} \phi_{a}\left(\psi_{j} \varepsilon \psi_{k}\right)=y_{j k}^{a} \phi_{a}\left(\psi^{j} \varepsilon \psi^{k}\right) \quad \text { and } \quad \mathrm{m}^{j k}\left(\psi_{j} \varepsilon \psi_{k}\right)=\mathrm{m}_{j k}\left(\psi^{j} \varepsilon \psi^{k}\right)
$$

with raised fermionic indices denote the conjugation of Weyl components. This distinction is convenient to ensure that index contractions as in $\mathrm{m}^{i j} \mathrm{~m}_{j k}$ are due to the propagation of a fermion $\left\langle\psi_{j} \psi^{j}\right\rangle$. Since $\psi_{i}$ already contains all Weyl fermions as well as their conjugates, $\psi^{i}$ has the same degrees of freedom. Raising and lowering of fermionic indices merely represents a pairwise permutation of components in $\psi$, such that (2.2) holds. Note that our notation is very close to the one suggested in [12], where each component of a fermion $\Psi$ contains both a Weyl spinor and its conjugate. Raising and lowering of fermionic indices in [12] is made explicit by multiplication with the Pauli matrices $\sigma_{1}$.

As the scalars are expressed in terms of real components, the raised or lowered position of their indices has no significance. ${ }^{1}$ In the following, we suppress fermionic indices for convenience, where it is understood that, e.g., $y^{a} y^{b} \mathrm{~m} y^{c}=y^{a i j} y_{j k}^{b} \mathrm{~m}^{k l} y_{l r}^{c}$, and the additional abbreviation $y^{a} y^{b} y^{c} y^{d} \ldots=y^{a b c d \ldots}$ is utilised.

Connecting to the notation of e.g. [1-3], where Weyl fermions $\Psi_{i}$ and their conjugates $\Psi_{i}^{*}$ are treated separately

$$
\begin{aligned}
\mathcal{L}= & i \Psi_{j}^{\dagger} \bar{\sigma}^{\mu} \partial_{\mu} \Psi_{j}-\frac{1}{2} Y_{j k}^{a} \phi_{a}\left(\Psi_{j} \varepsilon \Psi_{k}\right)-\frac{1}{2} Y_{j k}^{\dagger}{ }_{j a} \phi_{a}\left(\Psi_{j}^{*} \varepsilon \Psi_{k}^{*}\right) \\
& -\frac{1}{2} \mathrm{~m}_{j k}\left(\Psi_{j} \varepsilon \Psi_{k}\right)-\frac{1}{2} \mathrm{~m}_{j k}^{\dagger}\left(\Psi_{j}^{*} \varepsilon \Psi_{k}^{*}\right)+\ldots,
\end{aligned}
$$

the relation

$$
\operatorname{tr}\left(y^{a} y^{b} y^{c} y^{d} \ldots\right)=\operatorname{tr}\left(Y^{a} Y^{\dagger b} Y^{c} Y^{\dagger d} \ldots\right)+\operatorname{tr}\left(Y^{\dagger a} Y^{b} Y^{\dagger c} Y^{d} \ldots\right)
$$

holds, while untraced products are simply expressed via $Y^{a} Y^{\dagger b} Y^{c} \ldots=y^{a b c \ldots}$.

Through the course of this paper, we determine the RG evolution for general QFTs without gauge interactions at three-loop in perturbation theory. At this loop order, results depend on the regularisation and renormalisation procedure, for which we employ dimensional regularisation (DREG) [37, 38] and the $\overline{\mathrm{MS}}$ scheme $[39,40]$. Due to this choice, there is also an inherent ambiguity in the treatment of $\gamma_{5}$, which is discussed in the next section. As all gaugeless and renormalisable QFTs can be mapped onto our template (2.1), we aim to determine $\beta$ - and $\gamma$-functions for its generalised couplings and fields. Overall, we will proceed to formulate our ansätze for three-loop RGEs in terms of contractions of the dimensionless couplings $\lambda_{a b c d}$ and $y^{a}$ only, and employ the dummy field trick [4, 5, 41] to extract $\beta$-functions for $h_{a b c}, m_{a b}^{2}$ and $\mathrm{m}^{i j}$. To facilitate the evaluation, a modified version of ARGES [15] is employed.

\footnotetext{
${ }^{1}$ For instance, quartic couplings $\lambda_{a b c d}$ appear with lowered, while Yukawas $y^{a}$ with raised scalar indices in this work, which has no other significance but being a style preferred by the author.
} 


\section{$3 \quad$ Handling of $\gamma_{5}$}

The DREG procedure prescribes the evaluation of loop integrals in $d=4-2 \epsilon$ instead of four dimensions. However, this is incompatible with the spinor algebra of Dirac matrices $\gamma^{\mu}[42]$, as the definition

$$
\gamma_{5}=\frac{i}{24} \epsilon_{\mu \nu \rho \sigma} \gamma^{\mu} \gamma^{\nu} \gamma^{\rho} \gamma^{\sigma} \quad \text { with } \quad \epsilon_{0123}=-\epsilon^{0123}=1
$$

being the Levi-Civita symbol, is closely tied to four dimensions. Different approaches to reconcile the $\gamma_{5}$ problem — see [17] for a review and [43] for a recent list of works — in general lead to ambiguous computational results; this includes renormalisation group equations, see e.g. [44, 45]. The source of these ambiguities is the occurrence of the expression

$$
\operatorname{tr}\left[\gamma^{\mu} \gamma^{\nu} \gamma^{\rho} \gamma^{\sigma} \gamma_{5}\right]= \begin{cases}4 i \epsilon^{\mu \nu \rho \sigma} & \text { for } d=4, \\ 0 & \text { naïvely in } d \text { dimensions with }\left\{\gamma^{\mu}, \gamma_{5}\right\}=0\end{cases}
$$

in loop corrections. In [28, 46], a semi-naïve scheme has been chosen that renders the ambiguity evanescent $(\propto d-4)$ and hence drop out when computing three-loop Yukawa $\beta$-functions. This scheme has also been employed in [27, 32-34], which serve as input in this work. At four-loop order, the approach is not sufficient, but contributions affected by the $\gamma_{5}$ problem in $[12,19,24]$ have been fixed using Weyl consistency conditions.

In our setup, tensor structures that explicitly distinguish the chiralities of the included fermion lines have to be considered in the ansatz in order to account for possible $\gamma_{5}$ corrections. This could be achieved by inserting the quantity $\chi_{j}{ }_{j}= \pm \delta^{i}{ }_{j}$ on fermion lines, providing opposite signs for left- and right-chiral fermions. Obviously, this is a direct adaptation of $\gamma_{5}$ into the language of Weyl components. However, due to the absence of gauge interactions, there are actually no $\gamma_{5}$ ambiguities in this work. Following the argumentation in $[12,19,28,46]$, a non-vanishing term (3.2) only occurs if there is a combination of four independent momenta and/or Lorentz indices. All RGEs are independent of external momenta, which can be set to zero in the final result. As gauge fields are not present in our work, four independent loop momenta are required to generate an ambiguity from (3.2). This, however, cannot occur at three-loop order as investigated here, but would become relevant at four-loop. In fact, all ambiguous three-loop terms in the general Yukawa $\beta$ function, as well as scalar and fermion field anomalous dimensions, have been identified in $[12,19]$ to contain gauge interactions. Consequently, the $\gamma_{5}$ issue can be neglected both in our ansatz as well as in the input data we use, as the gauge sector will be projected out.

\section{Scalar anomalous dimension}

The canonical renormalisation procedure of the scalar fields entails the introduction of symmetric field strength renormalisation matrices $Z_{a b}^{\phi}=Z_{b a}^{\phi}$ via $\phi_{a} \mapsto \sqrt{Z^{\phi}} a b \phi_{b}$ in (2.1). The running of this quantity under a change of the renormalisation scale $\mu$ is then encoded in the scalar field anomalous dimension

$$
\gamma_{a b}^{\phi}=\frac{\mathrm{d} \sqrt{Z^{\phi}} a c}{\mathrm{~d} \ln \mu}\left(\sqrt{Z^{\phi}}\right)_{c b}^{-1}=\sum_{n=1}^{\infty} \frac{\gamma_{a b}^{\phi, n \ell}}{(4 \pi)^{2 n}} .
$$




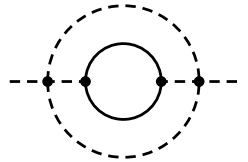

(1)

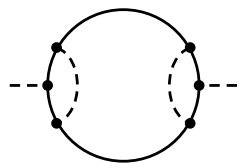

(6)

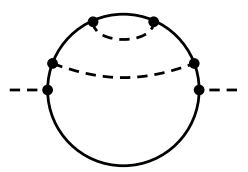

(11)

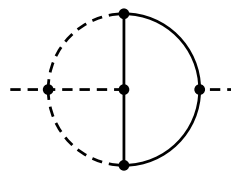

(2)

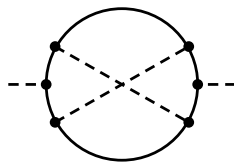

(7)

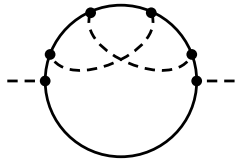

(12)

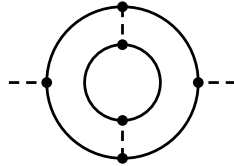

(3)

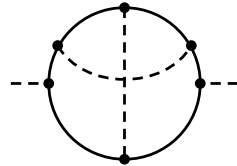

(8)

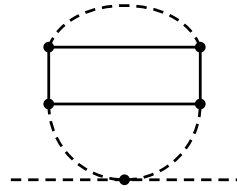

(13)

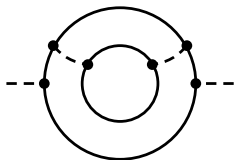

(4)

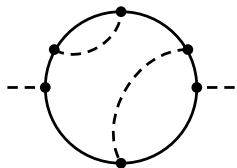

(9)

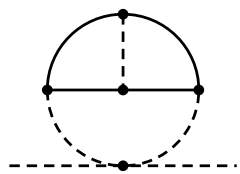

(14)

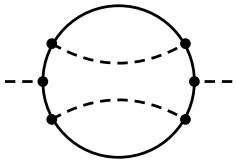

(5)

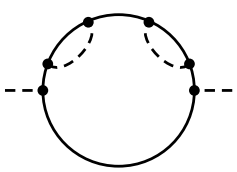

(10)

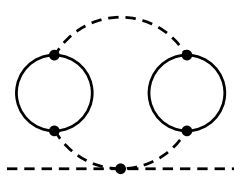

(15)

Figure 1. Fermionic (solid lines) and scalar (dashed lines) diagrams potentially giving rise to three-loop corrections of scalar field anomalous dimensions. One additional diagram providing a purely scalar contribution is not shown.

The one- and two-loop results $\gamma^{\phi, 1 \ell}$ and $\gamma^{\phi, 2 \ell}$ of this quantity are known for arbitrary renormalisable QFTs $[1,4]$, and the pure scalar (quartic) three-loop contribution to $\gamma^{\phi, 3 \ell}$ has been found in $[22,23,25,26]$. Extending this progress with an arbitrary Yukawa sector, we find 15 additional contractions, listed in figure 1. Among those are also (2) and (9) which are manifestly asymmetric under exchange of external legs. While $Z_{a b}^{\phi}=Z_{b a}^{\phi}$, the square root $\sqrt{Z^{\phi}}$ ab is only symmetric up to an arbitrary an orthogonal transformation of the scalar field species, which is translated into a potential asymmetry of $\gamma^{\phi}$ by virtue of (4.1). Such rotations in the basis of scalar fields correspond to transformations of running couplings, which drops out in physical observables and are absorbed by the antisymmetric parts of the field anomalous dimensions [27, 32, 47]. We use [27] to fix all open coefficients, where anomalous dimensions are chosen completely symmetric. This yields

$$
\begin{aligned}
\gamma_{a b}^{\phi, 3 \ell}= & -\frac{1}{16} \lambda_{a c d e} \lambda_{d e f g} \lambda_{b c f g}-\frac{5}{32} \lambda_{a c d e} \lambda_{b c d f} \operatorname{tr}\left(y^{e f}\right) \\
& +\frac{5}{8}\left[\lambda_{a c d e} \operatorname{tr}\left(y^{b c d e}\right)+\operatorname{tr}\left(y^{a c d e}\right) \lambda_{b c d e}\right]+\left[\operatorname{tr}\left(y^{a b c d}\right)+\frac{9}{16} \operatorname{tr}\left(y^{a c b d}\right)\right] \operatorname{tr}\left(y^{c d}\right) \\
& -\frac{3}{16} \operatorname{tr}\left(y^{a b c c d d}\right)+\frac{5}{16} \operatorname{tr}\left(y^{a b c d d c}\right)-\frac{3}{8} \operatorname{tr}\left(y^{a b c d c d}\right)+\frac{7}{16}\left[\operatorname{tr}\left(y^{a c b c d d}\right)+\operatorname{tr}\left(y^{b c a c d d}\right)\right] \\
& -\frac{3}{4} \operatorname{tr}\left(y^{a c b d c d}\right)+\frac{1}{32} \operatorname{tr}\left(y^{a c c b d d}\right)+\frac{7}{4} \operatorname{tr}\left(y^{a c d b d c}\right)+\left[\frac{3}{2} \zeta_{3}-1\right] \operatorname{tr}\left(y^{a c d b c d}\right)
\end{aligned}
$$

employing the abbreviation $y^{a b c d \ldots}=y^{a} y^{b} y^{c} y^{d} \ldots$ and $\zeta_{3} \approx 1.202$ is Apéry's constant. It is found that the graphs (13)-(15) in figure 1 do not contribute to the anomalous dimension, which can be readily understood from the momentum flow. 


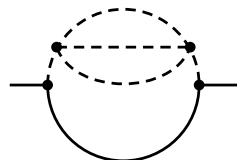

(1)

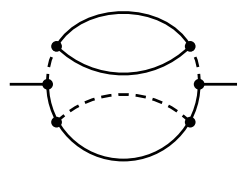

(6)

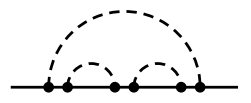

(11)

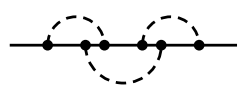

(16)

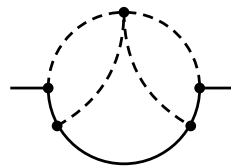

(2)

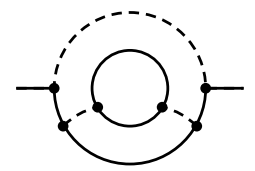

(7)

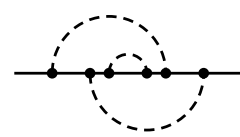

(12)

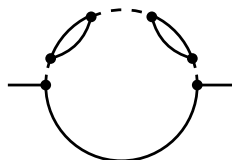

(3)

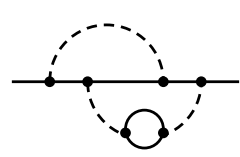

(8)

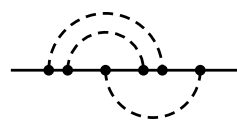

(13)

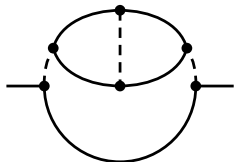

(4)

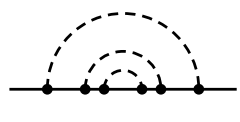

(9)

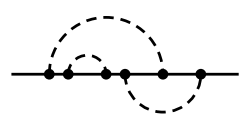

(14)

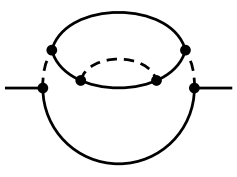

(5)

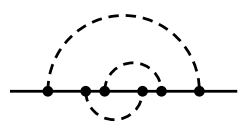

(10)

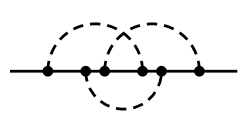

(15)

Figure 2. Three-loop diagrams giving contributions to the fermion field anomalous dimensions, containing Yukawa and quartic interactions of fermions (solid lines) and scalar fields (dashed lines).

\section{Fermion anomalous dimension}

Similar to the scalar case, fermions are renormalised by introducing a field strength matrix $\psi_{i} \mapsto\left(\sqrt{Z^{\psi}}\right)_{i}{ }^{j} \psi_{j}$. Dropping the indices for convenience, the fermion anomalous dimension is defined via

$$
\gamma^{\psi}=\frac{\mathrm{d} \sqrt{Z^{\psi}}}{\mathrm{d} \ln \mu}\left(\sqrt{Z^{\psi}}\right)^{-1}=\sum_{n=1}^{\infty} \frac{\gamma^{\psi, n \ell}}{(4 \pi)^{2 n}}
$$

Again, the leading and next-to-leading orders are available in full generality [1, 4]. Diagrams contributing to the three-loop expression $\gamma^{\psi, 3 \ell}$ containing Yukawa and quartic interactions are listed in figure 2. Here, (8), (13) and (14) are not symmetric under permutation of their external legs. Similar to the scalar case $\sqrt{Z^{\phi}}$, these are linked to a potential ambiguity of an unitary transformation for fermionic field multiplets in the definition of $\sqrt{Z^{\psi}}$, which is unphysical, see [27, 32, 47] for a more detailed discussion. In this work, we use [27] to determine all parameters, electing anomalous dimensions to be hermitian. This fixes the 
ambiguous diagrams in figure 2 to be symmetrised, thus leading to the general expression

$$
\begin{aligned}
\gamma^{\psi, 3 \ell}= & -\frac{11}{96} \lambda_{a c d e} \lambda_{b c d e}\left(y^{a b}\right)+\lambda_{a b c d}\left(y^{a b c d}\right) \\
& -\frac{3}{32} \operatorname{tr}\left(y^{a c}\right) \operatorname{tr}\left(y^{b c}\right)\left(y^{a b}\right)+\operatorname{tr}\left(y^{a b c c}+\frac{1}{2} y^{a c b c}\right)\left(y^{a b}\right) \\
& -\frac{3}{32} \operatorname{tr}\left(y^{a b}\right)\left[\frac{4}{3} y^{a c c b}-3 y^{c a b c}+y^{a c b c}+y^{c a c b}\right] \\
& -\frac{5}{32}\left(y^{a b b c c a}\right)+\frac{1}{16}\left(y^{a b c c b a}\right)-\frac{5}{16}\left(y^{a b c b c a}\right)+\frac{1}{4}\left(y^{a b c c a b}\right) \\
& +\frac{3}{32}\left(y^{a b b c a c}+y^{a b a c c b}\right)+\left[\frac{3}{2} \zeta_{3}-1\right]\left(y^{a b c a b c}\right)+\frac{1}{2}\left(y^{a b a c b c}\right) .
\end{aligned}
$$

In particular, this means that the two diagrams depicted by figure 2 (13) do not contribute to the fermion anomalous dimension, while (8) and (14) are symmetrised.

\section{Yukawa interaction and fermion masses}

The $\beta$-functions of the renormalised Yukawa couplings $y^{a i j}$ are defined via

$$
\beta_{y^{a}}=\frac{\mathrm{d} y^{a}}{\mathrm{~d} \ln \mu}=\sum_{n=1}^{\infty}(4 \pi)^{-2 n} \beta_{y^{a}}^{n \ell},
$$

and template formulas for the one- and two-loop expressions can be found in $[2,4,5,12]$. The three-loop part $\beta_{y^{a}}^{3 \ell}$ consists of fermionic (5.2) and scalar leg contributions (4.2), as well as proper Yukawa vertex corrections. In our setup without gauge interactions, the latter are listed in figure 3 and give rise to 52 open parameters, considering that the vertex is symmetric under permutation of its fermionic legs. This ansatz has been cross-checked 


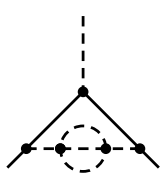

(1)

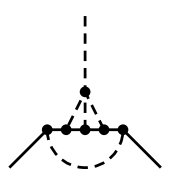

(8)

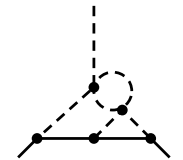

(2)

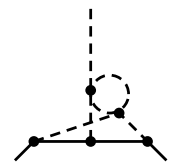

(3)

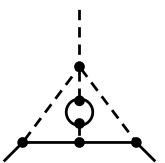

(4)

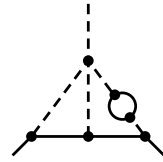

(5)

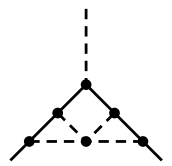

(6)

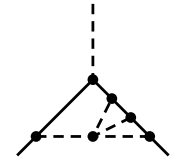

(7)

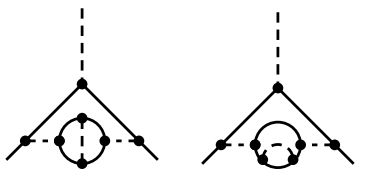

(15)

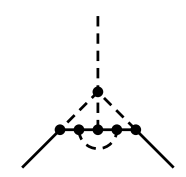

(9)

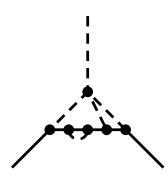

(10)

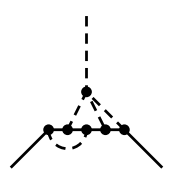

(11)

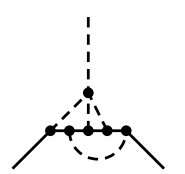

(12)

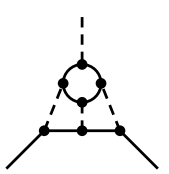

(13)

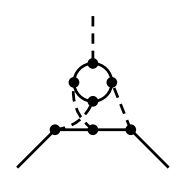

(14)
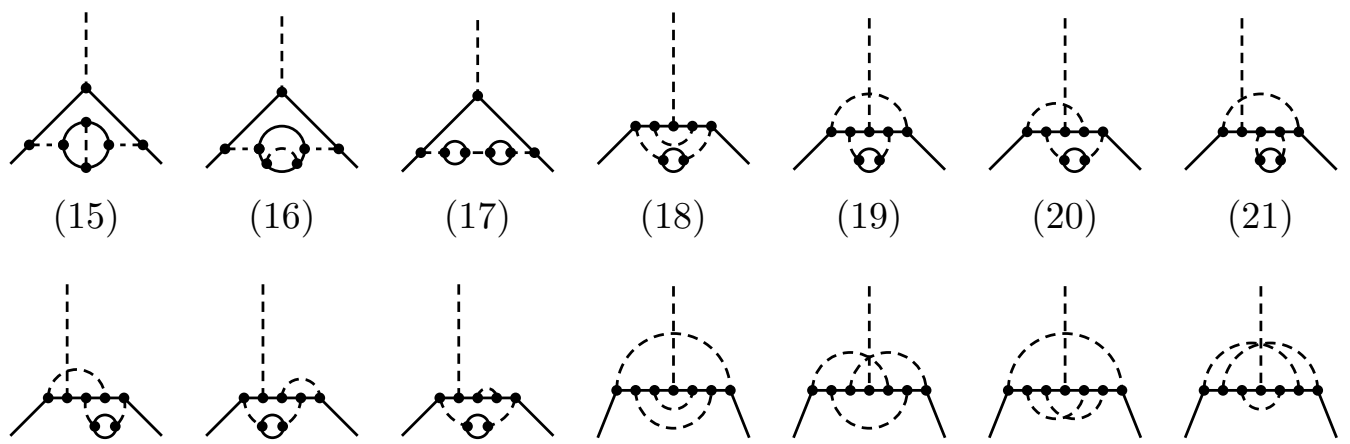

(17)

(18)

(19)

(20)

(21)
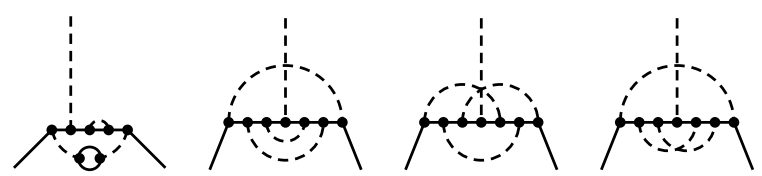

(27)

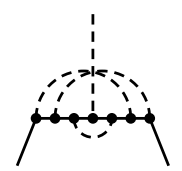

(22)

(23)

(24)

(25)

(26)
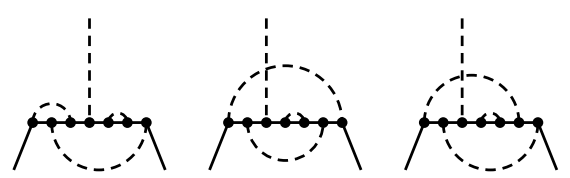

(28)
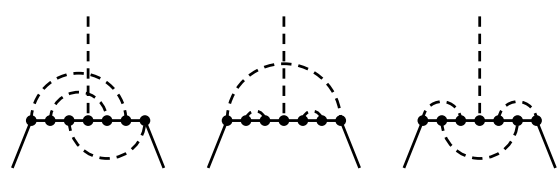

(32)

(33)

(34)

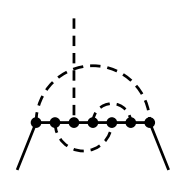

(35)
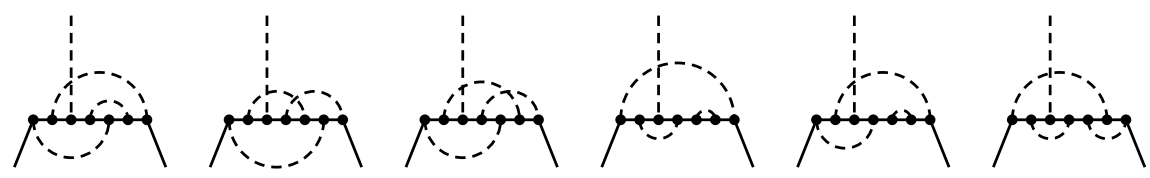

(41)

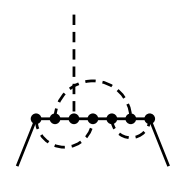

(36)

(37)

(38)

(39)

(40)

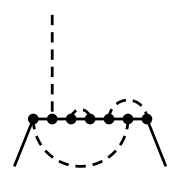

(42)
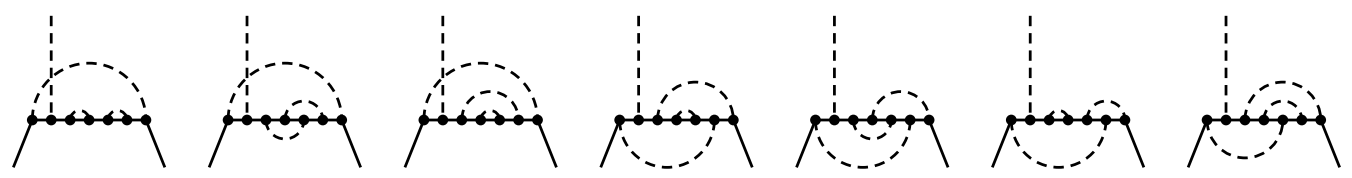

(43)

(44)

(45)

(46)

(47)

(48)

(49)
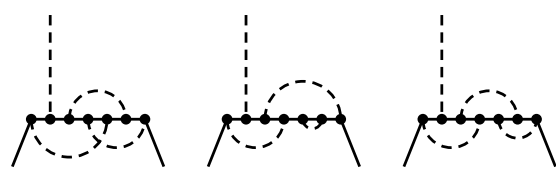

(50)

(51)

(52)

Figure 3. Yukawa proper vertex diagrams at three-loop order, including scalar (dashed lines) and fermionic propagators (solid lines). 
with diagram list provided with [12]. Using [27], the three-loop expression

$$
\begin{aligned}
& \beta_{y^{a}}^{3 \ell}=\gamma_{a b}^{\phi, 3 \ell} y^{b}+\gamma^{\psi, 3 \ell} y^{a}+y^{a}\left(\gamma^{\psi, 3 \ell}\right)^{\top}-\frac{3}{8} \lambda_{b \operatorname{def}} \lambda_{c d e f}\left(y^{b a c}\right) \\
& +\frac{1}{2}\left[\lambda_{a e b f} \lambda_{b f c d}+\lambda_{a b c d} \operatorname{tr}\left(y^{b e}\right)\right]\left[y^{c d e}+y^{e c d}+3 y^{c e d}\right] \\
& +\lambda_{b c d e}\left(2 y^{b c a d e}+3 y^{b a c d e}+3 y^{b c d a e}\right) \\
& +\lambda_{a b c d}\left[5 y^{\text {ebcde }}+3 y^{\text {beced }}+\frac{1}{2} y^{\text {beecd }}+\frac{1}{2} y^{\text {bceed }}\right] \\
& +\lambda_{a b c d}\left[-y^{e b e c d}-y^{b c e d e}+2 y^{e b c e d}+2 y^{b e c d e}\right]-\frac{1}{2} \operatorname{tr}\left(y^{b d}\right) \operatorname{tr}\left(y^{d c}\right)\left(y^{b a c}\right) \\
& +2\left[3 \zeta_{3}-2\right] \operatorname{tr}\left(y^{a b c d}\right)\left[y^{b d c}+y^{c b d}\right]+\left[\frac{5}{4} \operatorname{tr}\left(y^{b d c d}\right)+\frac{25}{8} \operatorname{tr}\left(y^{b c d d}\right)\right]\left(y^{b a c}\right) \\
& +\operatorname{tr}\left(y^{b c}\right)\left[2 y^{d b a c d}-y^{b d a d c}-\frac{1}{2} y^{b d a c d}-\frac{1}{2} y^{d b a d c}+\frac{25}{16} y^{d b c a d}+\frac{25}{16} y^{d a b c d}\right] \\
& +\frac{3}{2} \operatorname{tr}\left(y^{b c}\right)\left[y^{b d c a d}+y^{d a b d c}-y^{d b d a c}-y^{b a d c d}-\frac{1}{3} y^{b d d a c}-\frac{1}{3} y^{b a d d c}\right] \\
& +4 y^{b c d a d c b}-3 y^{b c d a d b c}+\left[6 \zeta_{3}-5\right] y^{b c d a c d b}+\left[6 \zeta_{3}-2\right]\left[y^{b c d a c d b}+y^{b c d a b d c}\right] \\
& +2 y^{b c d a b c d}-2 y^{b c b a d c d}-\frac{1}{2} y^{b c c a d d b}-\frac{3}{2}\left[y^{b c b a d d c}+y^{b d d a c b c}\right] \\
& +y^{b c}\left(y^{a d d}+y^{d d a}\right)\left(2 y^{c b}-\frac{3}{2} y^{b c}\right)-y^{b c}\left(y^{a d c}+y^{d c a}\right) y^{d b} \\
& +\left[6 \zeta_{3}-3\right]\left(y^{b c a d b d c}+y^{b d c d a b c}\right)-4\left(y^{b c a d c b d}+y^{d b c d a c b}\right)-y^{b c a c d d b}-y^{b d d c a c b} \\
& +\left[6 \zeta_{3}-2\right]\left(y^{b c a d b c d}+y^{d b c d a b c}\right)-\frac{1}{2}\left(y^{b c a b d d c}+y^{b d d c a b c}\right)-3\left(y^{b c a c d b d}+y^{b d b c a c d}\right) \\
& +y^{b c}\left(y^{a b d}+y^{b d a}\right) y^{c d}-\frac{1}{2}\left(y^{b a c c d d b}+y^{b c c d d a b}\right)-y^{b a c d c d b}-y^{b c d c d a b} \\
& +\frac{7}{16}\left(y^{b a c d d c b}+y^{b c d d c a b}\right)+\frac{1}{2}\left(y^{b a c d d b c}+y^{b c d d b a c}\right)-2\left(y^{b a d c d b c}+y^{b c d b d a c}\right) \\
& -\frac{3}{2}\left(y^{b a d d c b c}+y^{b c b d d a c}\right)+y^{b a c d b d c}+y^{b d c d b a c}+3\left[2 \zeta_{3}-1\right]\left(y^{b a d c b d c}+y^{b d c b d a c}\right) \\
& +\frac{3}{2}\left(y^{b a c b d d c}+y^{b d d c b a c}\right)+2\left(y^{b a d b c d c}+y^{b d b c d a c}\right)
\end{aligned}
$$

is obtained. This automatically provides the renormalisation group evolution for the fermion mass term $\beta_{\mathrm{m}}^{3 \ell}$ after the replacement

$$
\gamma_{a b}^{\phi, 3 \ell} y^{b} \mapsto 0, \quad y^{a} \mapsto \mathrm{m}, \quad \lambda_{a b c d} \mapsto h_{b c d}
$$

in (6.2). Furthermore, the result (6.2) has been cross-checked against [21, 28, 32, 46]. For convenience, we have collected all coefficients of $\beta_{y}^{3 \ell}$ in the basis from [12] in appendix A.

\section{$7 \quad$ Scalar quartic interactions}

For scalar quartic interactions $\lambda_{a b c d}$, the $\beta$-functions

$$
\beta_{\lambda_{a b c d}}=\frac{\mathrm{d} \lambda_{a b c d}}{\mathrm{~d} \ln \mu}=\sum_{n=1}^{\infty}(4 \pi)^{-2 n} \beta_{\lambda_{a b c d} \ell}
$$




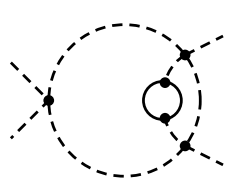

(1) $\times 6$

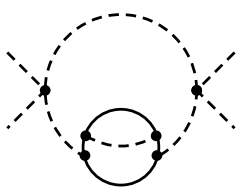

(6) $\times 3$

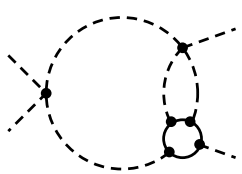

(11) $\times 12$

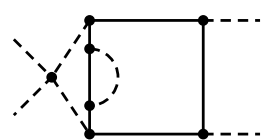

$(16) \times 6$

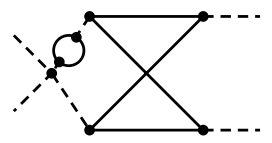

$(21) \times 6$

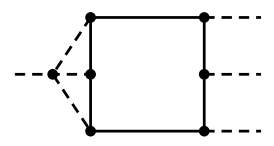

(26) $\times 12$

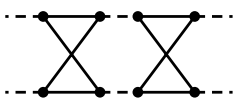

$(31) \times 3$

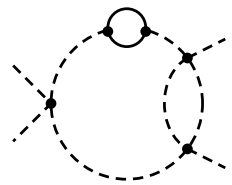

(2) $\times 12$

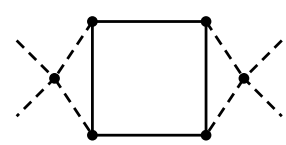

(7) $\times 3$

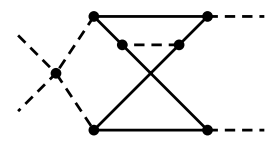

$(22) \times 6$

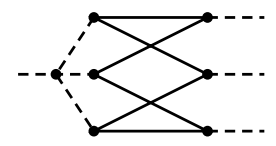

$(27) \times 4$
(8) $\times 3$

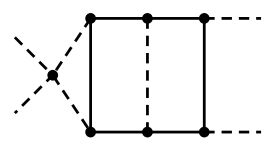

(13) $\times 6$

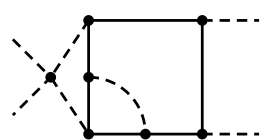

(18) $\times 12$
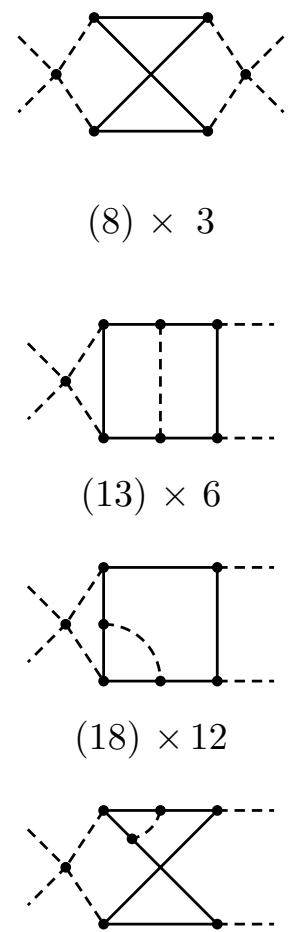

$(23) \times 6$

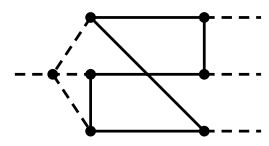

$(28) \times 24$

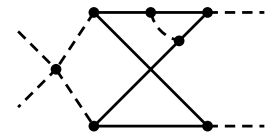

(24) $\times 12$

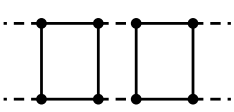

$(29) \times 6$

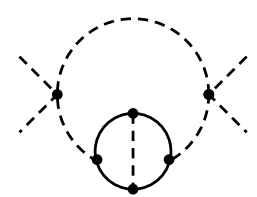

(5) $\times 3$
(9) $\times 12$

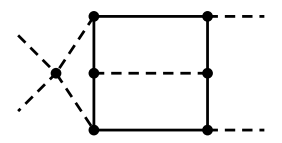

(14) $\times 6$

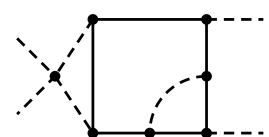

(19) $\times 12$
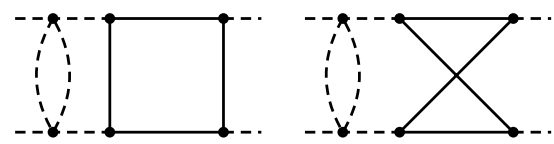

(10) $\times 12$

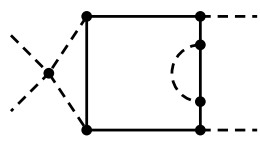

$(15) \times 6$

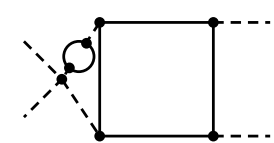

(20) $\times 12$

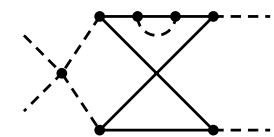

$(25) \times 12$

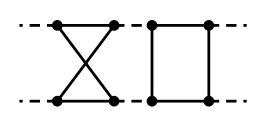

$(30) \times 6$

Figure 4. Three-loop diagrams contributing to the scalar quartic vertex renormalisation, containing both quartic and Yukawa vertices. Solid [dashed] lines correspond to fermionic [scalar] propagators. The number of inequivalent permutations of the external legs for each diagram is also indicated. 


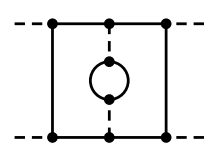

$(32) \times 6$

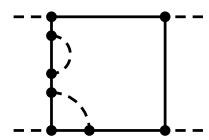

$(38) \times 24$

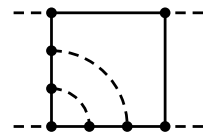

$(44) \times 12$

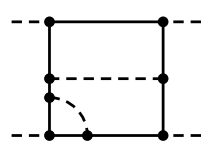

$(50) \times 24$

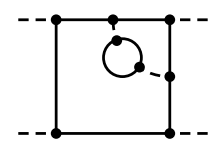

(33) $\times 12$

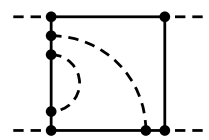

(39) $\times 24$

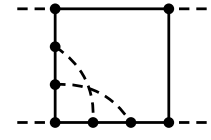

$(45) \times 12$

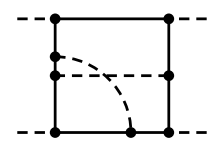

(51) $\times 24$

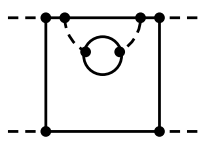

(34) $\times 12$

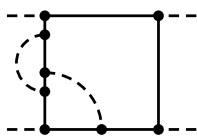

$(40) \times 24$

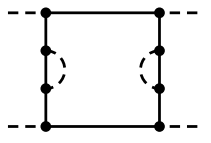

$(46) \times 6$

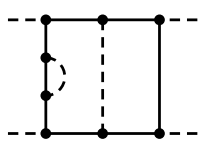

$(52) \times 12$

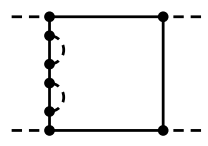

$(35) \times 12$

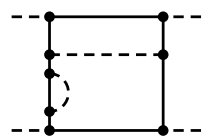

$(41) \times 24$

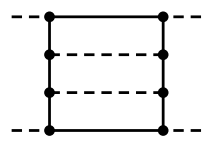

$(47) \times 6$

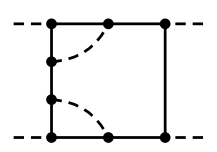

$(53) \times 12$

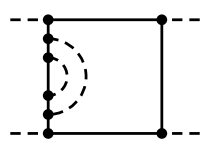

(36) $\times 12$

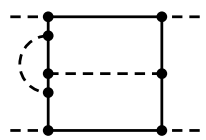

(42) $\times 12$

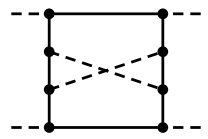

$(48) \times 6$

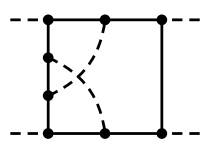

(54) $\times 12$

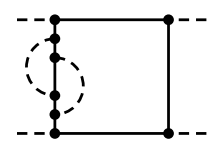

$(37) \times 12$

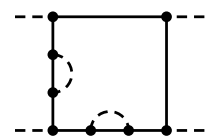

(43) $\times 12$

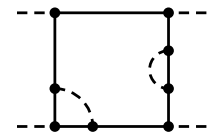

$(49) \times 24$

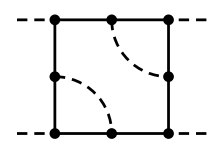

$(55) \times 6$

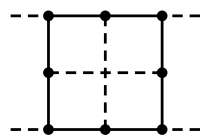

$(56) \times 3$

Figure 5. Three-loop diagrams contributing to the scalar quartic vertex renormalisation, containing only Yukawa couplings with a single fermion loop (solid lines) and scalar propagators (dashed lines) propagators. The number of inequivalent permutations of external legs is listed below each diagram.

have been computed for general QFTs at two-loop order [3-5, 12], and will now be extended to three-loop in the gaugeless limit via the ansatz (2.1). These expressions contain scalar leg corrections (4.2) which have been already determined. Moreover, the expression features proper scalar quartic vertex corrections that reflect the overall symmetrisation of the quartic interaction terms. These are listed in figure 4 and figure 5 , not including the six pure quartic contributions given in $[22,23,25]$. Thus, 56 open parameters are implied. Three-loop SM results [28-32] as well as $\beta$ - and $\gamma$-functions in Gross-Neveu-Yukawa theories, in particular chiral Ising, XY and Heisenberg models [33, 34], can be utilised for matching unknown coefficients. Additional input data can be extracted when comparing to $\mathcal{N}=1$ supersymmetric QFTs as detailed in appendix B. Overall, from the 56 open parameters, 29 coefficients can be determined directly, and 14 supplementary conditions apply, leaving only 13 pieces of information missing.

We are currently unaware of pre-existing calculation that would fix these open coefficients. No data from the THDM is available, as [27] does not include three-loop quartic $\beta$ 
functions, while [48] lacks the Yukawa contributions in question. Moreover, no conditions in [12] apply to coefficients at this loop order. Similarily, no three-loop quartic RGEs are included in [21] either.

Concretely, we obtain the three-loop $\beta$-function of the scalar quartic as

$$
\begin{aligned}
\beta_{\lambda_{a b c d}}^{3 \ell}= & \gamma_{a e}^{\phi, 3 \ell} \lambda_{e b c d}+\gamma_{b e}^{\phi, 3 \ell} \lambda_{a e c d}+\gamma_{c e}^{\phi, 3 \ell} \lambda_{a b e d}+\gamma_{d e}^{\phi, 3 \ell} \lambda_{a b c e} \\
& +\beta_{a b c d}^{\lambda: \lambda^{4}}+\beta_{a b c d}^{\lambda: \lambda^{3} y^{2}}+\beta_{a b c d}^{\lambda: \lambda^{2} y^{4}}+\beta_{a b c d}^{\lambda: \lambda y^{6}}+\beta_{a b c d}^{\lambda: y^{8}}
\end{aligned}
$$

where the pure scalar part has been computed earlier $[22,23,25]$ and reads

$$
\begin{aligned}
\beta_{a b c d}^{\lambda: \lambda^{4}=} & 12 \zeta_{3} \lambda_{a e f g} \lambda_{b e h i} \lambda_{c f h j} \lambda_{d g i j}-\frac{1}{2}\left[\lambda_{a e f g} \lambda_{b f g h} \lambda_{c e i j} \lambda_{d h i j}+5 \text { perm. }\right] \\
& +\frac{1}{2}\left[\lambda_{a b e f} \lambda_{e g i j} \lambda_{f h i j} \lambda_{c d g h}+2 \text { perm. }\right]-\frac{3}{8}\left[\lambda_{a b e f} \lambda_{e h i j} \lambda_{g h i j} \lambda_{c d f g}+2 \text { perm. }\right] \\
& -\frac{1}{2}\left[\lambda_{a b e f} \lambda_{c e g h} \lambda_{g h i j} \lambda_{d f i j}+5 \text { perm. }\right]+2\left[\lambda_{a b e f} \lambda_{c e g h} \lambda_{f g i j} \lambda_{d h i j}+11 \text { perm. }\right]
\end{aligned}
$$

Insertions of a single Yukawa bubble $\operatorname{tr}\left(y^{2}\right)$ give rise to the correction

$$
\begin{aligned}
\beta_{a b c d}^{\lambda: \lambda^{3} y^{2}}= & 2\left[\lambda_{\text {abef }} \lambda_{c e g h} \lambda_{d f g i} \operatorname{tr}\left(y^{h i}\right)+5 \text { perm. }\right] \\
& -\frac{1}{2}\left[\lambda_{\text {abef }} \lambda_{c e g h} \lambda_{d g h i} \operatorname{tr}\left(y^{f i}\right)+11 \text { perm. }\right] .
\end{aligned}
$$

Further, $\lambda^{2} \operatorname{tr}\left(y^{2}\right) \operatorname{tr}\left(y^{2}\right)$ and $\lambda^{2} \operatorname{tr}\left(y^{4}\right)$ terms contribute to the $\beta$-function via

$$
\begin{aligned}
\beta_{a b c d}^{\lambda: \lambda^{2} y^{4}}= & -\frac{1}{4}\left[\lambda_{\text {abef }} \lambda_{\text {cdgh }}+2 \text { perm. }\right]\left[\operatorname{tr}\left(y^{e g}\right) \operatorname{tr}\left(y^{f h}\right)+2 \delta^{e g} \operatorname{tr}\left(y^{f i}\right) \operatorname{tr}\left(y^{i h}\right)\right] \\
& +\left[\lambda_{\text {abef }} \lambda_{\text {cdgh }}+2 \text { perm. }\right]\left[\left(3 \zeta_{3}-1\right) \operatorname{tr}\left(y^{e f f h}\right)+2 \operatorname{tr}\left(y^{e f g h}\right)\right] \\
& +\left[\lambda_{\text {abef }} \lambda_{\text {cdeg }}+2 \text { perm. }\right]\left[\frac{25}{8} \operatorname{tr}\left(y^{f g h h}\right)+\frac{5}{4} \operatorname{tr}\left(y^{f h g h}\right)\right] \\
& +\left[\lambda_{\text {aefg }} \lambda_{\text {befh }}\left(\operatorname{tr}\left(y^{c g d h}\right)+3 \operatorname{tr}\left(y^{c d g h}\right)\right)+11 \text { perm. }\right] \\
& +2\left[\lambda_{\text {abef }} \lambda_{\text {cegh }}\left(\operatorname{tr}\left(y^{\text {dfgh }}\right)+\left(3 \zeta_{3}-2\right) \operatorname{tr}\left(y^{\text {dgfh }}\right)\right)+11 \text { perm. }\right] .
\end{aligned}
$$

While terms of the form $\lambda \operatorname{tr}\left(y^{2}\right)^{3}$ are absent, $\lambda \operatorname{tr}\left(y^{2}\right) \operatorname{tr}\left(y^{4}\right)$ and $\lambda \operatorname{tr}\left(y^{6}\right)$ contractions read

$$
\begin{aligned}
\beta_{\text {abcd }}^{\lambda: \lambda y^{6}}= & 3\left[\lambda_{\text {abef }} \operatorname{tr}\left(y^{f g}\right) \operatorname{tr}\left(y^{\text {gecd }}\right)+11 \text { perm. }\right] \\
& +2\left[\lambda_{\text {abef }} \operatorname{tr}\left(y^{f g}\right) \operatorname{tr}\left(y^{g c e d}\right)+5 \text { perm. }\right] \\
& -\left[\lambda_{\text {abef }}\left(4 \operatorname{tr}\left(y^{\text {ecfdgg }}\right)+3 \operatorname{tr}\left(y^{\text {cdggef }}\right)\right)+11 \text { perm. }\right] \\
& +\left[\lambda_{\text {abef }}\left(c_{(13)} \operatorname{tr}\left(y^{\text {cdgefg }}\right)-10 \operatorname{tr}\left(y^{\text {cgdegf }}\right)\right)+5 \text { perm. }\right] \\
& -\left[\lambda_{\text {abef }}\left(c_{(15)} \operatorname{tr}\left(y^{\text {cggdef }}\right)-\left(4-\frac{3}{2} \zeta_{3}\right) \operatorname{tr}\left(y^{\text {cdeggf }}\right)\right)+5 \text { perm. }\right] \\
& +\left[\lambda_{\text {abef }} \operatorname{tr}\left(c_{(18)} y^{\text {cdgegf }}+c_{(19)} y^{\text {cgdgef }}\right)+11 \text { perm. }\right] \\
& -\left(17-\frac{15}{2} \zeta_{3}+2 c_{(13)}+2 c_{(15)}\right)\left[\lambda_{\text {abef }} \operatorname{tr}\left(y^{\text {cegdfg }}\right)+5 \text { perm. }\right]
\end{aligned}
$$




$$
\begin{aligned}
& +c_{(24)}\left[\lambda_{\text {abef }} \operatorname{tr}\left(y^{\text {cegdgf }}\right)+11 \text { perm. }\right] \\
& -\left[17-\frac{27}{2} \zeta_{3}+2 c_{(24)}\right]\left[\lambda_{\text {abef }} \operatorname{tr}\left(y^{\text {cgegdf }}\right)+5 \text { perm. }\right] \\
& -\left(\frac{23}{2}+6 \zeta_{3}+\frac{1}{2} c_{(13)}+\frac{3}{2} c_{(15)}\right)\left[\lambda_{\text {aefg }} \operatorname{tr}\left(y^{\text {bcdefg }}\right)+11 \text { perm. }\right] \\
& -\frac{3}{2}\left(3+19 \zeta_{3}+c_{(13)}+c_{(15)}\right)\left[\lambda_{\text {aefg }} \operatorname{tr}\left(y^{\text {becfdg }}\right)+3 \text { perm. }\right] \\
& +\left(2-9 \zeta_{3}+1+\frac{1}{2} c_{(13)}+c_{(15)}\right)\left[\lambda_{\text {aefg }} \operatorname{tr}\left(y^{\text {bcedf } g}\right)+23 \text { perm. }\right],
\end{aligned}
$$

where the coefficients corresponding to contractions figure 4 (13), (13), (18), (19) and (24) could not be determined completely, and are retained as $c_{(13)}, c_{(15)}, c_{(18)}, c_{(19)}$ and $c_{(24)}$. Finally, pure Yukawa terms $\operatorname{tr}\left(y^{4}\right) \operatorname{tr}\left(y^{4}\right), \operatorname{tr}\left(y^{2}\right) \operatorname{tr}\left(y^{6}\right)$ and $\operatorname{tr}\left(y^{8}\right)$ are given by

$$
\begin{aligned}
\beta_{a b c d}^{\lambda: y^{8}}= & -8\left[\operatorname{tr}\left(y^{\text {abef }}+\frac{3}{2} y^{\text {aebf }}\right) \operatorname{tr}\left(y^{\text {cdef }}\right)+5 \text { perm. }\right] \\
& -4\left[\operatorname{tr}\left(y^{\text {aebf }}\right) \operatorname{tr}\left(y^{\text {cedf }}\right)+2 \text { perm. }\right]-3\left[\operatorname{tr}\left(y^{\text {abecdf }}\right) \operatorname{tr}\left(y^{e f}\right)+5 \text { perm. }\right] \\
& -\operatorname{tr}\left(y^{e f}\right)\left[\frac{25}{8} \operatorname{tr}\left(y^{\text {abcdef }}\right)+4 \operatorname{tr}\left(y^{\text {abcedf }}\right)+11 \text { perm. }\right] \\
& +\left[\operatorname{tr}\left(y^{\text {abeecdff }}\right)+5 \text { perm. }\right]+\left[\operatorname{tr}\left(y^{\text {abcdeeff }}\right)+11 \text { perm. }\right] \\
& +\left[-\frac{7}{8} \operatorname{tr}\left(y^{\text {abcdeffe }}\right)+c_{(37)} \operatorname{tr}\left(y^{\text {abcdefef }}\right)+11 \text { perm. }\right] \\
& +\left[c_{(38)} \operatorname{tr}\left(y^{\text {affebecd }}\right)+c_{(39)} \operatorname{tr}\left(y^{\text {aeffbecd }}\right)+23 \text { perm. }\right] \\
& +\left[c_{(40)} \operatorname{tr}\left(y^{\text {aefebfcd }}\right)-\frac{3}{2} \zeta_{3} \operatorname{tr}\left(y^{\text {affebced }}\right)+23 \text { perm. }\right] \\
& +\left[c_{(42)} \operatorname{tr}\left(y^{\text {aefebcfd }}\right)+\operatorname{tr}\left(y^{\text {aeebffcd }}\right)+11 \text { perm. }\right] \\
& +\left[c_{(44)} \operatorname{tr}\left(y^{\text {aefbfecd }}\right)+\left(\frac{23}{2}-6 \zeta_{6}+\frac{1}{2} c_{(13)}+\frac{3}{2} c_{(15)}\right) \operatorname{tr}\left(y^{\text {aefbefcd }}\right)+11 \text { perm. }\right] \\
& +\left[-\left(8+2 c_{(13)}\right) \operatorname{tr}\left(y^{\text {aefbcfed }}\right)+c_{(48)} \operatorname{tr}\left(y^{\text {aefbcefd }}\right)+5 \text { perm. }\right] \\
& +\left[c_{(49)} \operatorname{tr}\left(y^{\text {aebecffd }}\right)+c_{(50)} \operatorname{tr}\left(y^{\text {aefbfced }}\right)+c_{(51)} \operatorname{tr}\left(y^{\text {aefbecfd }}\right)+23 \text { perm. }\right] \\
& -\left(4+c_{(15)}\right)\left[\operatorname{tr}\left(y^{\text {abecffde }}\right)+11 \text { perm. }\right] \\
& +\left[c_{(53)} \operatorname{tr}\left(y^{\text {abecefdf }}\right)+\left(3-\frac{3}{2} \zeta_{3}+c_{(13)}\right) \operatorname{tr}\left(y^{\text {abecfedf }}\right)+11 \text { perm. }\right] \\
& +c_{(55)}\left[\operatorname{tr}\left(y^{\text {aebfcedf }}\right)+2 \text { perm. }\right]+c_{(56)}\left[\operatorname{tr}\left(y^{\text {aebecfdf }}\right)+5 \text { perm. }\right] .
\end{aligned}
$$

while no one-particle-irreducible diagrams are formed by $\operatorname{tr}\left(y^{2}\right)^{4}$ bubbles. For notational 
compactness, we have not inserted the relations

$$
\begin{aligned}
c_{(19)}= & \frac{1}{2} c_{(13)}+\frac{1}{2} c_{(15)}-c_{(18)}-\frac{13}{2}+\frac{3}{2} \zeta_{3} \\
c_{(51)}= & -5-6 \zeta_{3}+c_{(13)}+c_{(15)}+c_{(13)}+2 c_{(37)}+c_{(39)} \\
& -c_{(40)}+c_{(42)}-c_{(44)}+3\left[c_{(38)}+c_{(49)}+c_{(50)}\right] \\
c_{(53)}= & 14\left(1-\frac{3}{2} \zeta_{3}-c_{(49)}-c_{(50)}\right)-8 c_{(37)}-12 c_{(38)}+2 c_{(44)} \\
& -4\left(c_{(13)}+c_{(15)}+c_{(39)}+c_{(42)}\right) \\
c_{(55)}= & -4\left(11+15 \zeta_{3}+c_{(13)}+c_{(15)}+c_{(37)}+c_{(42)}\right) \\
& -2 c_{(48)}-8\left(c_{(38)}+c_{(39)}+c_{(49)}+c_{(50)}\right) \\
c_{(56)}= & 21 \zeta_{3}-1+5\left(c_{(13)}+c_{(15)}\right)+8 c_{(37)}+12 c_{(38)} \\
& +4\left(c_{(39)}+c_{(42)}\right)-2 c_{(44)}+16\left(c_{(49)}+c_{(50)}\right)
\end{aligned}
$$

It may be possible to recover additional restrictions when comparing diagrams (38) with (49), (44) with (53) and (55), as well as (41) with (52) in figure 5 with a more sophisticated analysis of momentum integrals. Similar relations arise between diagrams (35), (43) and (46), as well as (3) and (4) in figure 4. However, this is insufficient to resolve all open parameters and will not be attempted here.

Just as in the Yukawa case, the renormalisation group equations for scalar cubic couplings $h_{a b c}$ and scalar masses $m_{a b}^{2}$ can be obtained from eq. (7.2)ff. using the dummy field method $[4,5,41]$. This entails substituting external scalar indices by dummy ones: $\varnothing$, corresponding to a classical, non-propagating and uncharged singlet. Using the substitutions

$$
\lambda_{a b c \varnothing}=h_{a b c}, \quad \lambda_{a b \varnothing \varnothing}=2 m_{a b}^{2}, \quad y^{\varnothing}=\mathrm{m}, \quad \gamma_{\varnothing e}^{\phi, 3 \ell}=0
$$

on both sides of eq. (7.2)ff. allows us to extract RGE parameters with positive canonical mass dimension. The last condition in (7.9) reflects that there is no field strength renormalisation for the dummy field. In general, the procedure will generate several distinct terms from permutations of the external indices in $\beta_{\lambda_{a b c d}}$. For instance, the correction

$$
\beta_{\lambda_{a b c d}}=\ldots+c_{(23)}\left[\lambda_{\text {abef }} \operatorname{tr}\left(y^{\text {cegdgf }}\right)+11 \text { perm. }\right]+\ldots
$$

from (7.6) will lead to a contribution to the scalar mass RG evolution that reads

$$
\begin{aligned}
\beta_{m_{a b}^{2}}= & \ldots+c_{(23)} \lambda_{a b e f} \operatorname{tr}\left(\mathrm{m} y^{e g} \mathrm{~m} y^{g f}\right)+c_{(23)} m_{e f}^{2} \operatorname{tr}\left(y^{\text {aegbgf }}+y^{\text {begagf }}\right) \\
& +c_{(23)} h_{a e f} \operatorname{tr}\left(\mathrm{m} y^{e g b g f}+y^{\text {beg }} \mathrm{m} y^{g f}\right)+c_{(23)} h_{b e f} \operatorname{tr}\left(\mathrm{m} y^{\text {egagf }}+y^{\text {aeg }} \mathrm{m} y^{g f}\right)
\end{aligned}
$$

which is significantly less compact. Hence, the overall expressions for $\beta_{h_{a b c}}$ and $\beta_{m_{a b}^{2}}$ are rather long compared to eq. (7.2)ff. and are omitted. For the sake of reducing the cross section of transcription errors alone, the author recommends to automate the application of the dummy field method onto the latter equations instead. 


\section{Example: Litim-Sannino model}

In this section, we apply the obtained results by computing the three-loop RGEs of the Litim-Sannino model $[49,50]$ with vanishing gauge couplings. The model is described in terms of the Lagrangian

$$
\begin{aligned}
\mathcal{L}= & \operatorname{tr}[\bar{\Psi} i \not \partial \Psi]+\operatorname{tr}\left[\partial_{\mu} H^{\dagger} \partial^{\mu} H\right]-m^{2} \operatorname{tr}\left[H^{\dagger} H\right] \\
& -y \operatorname{tr}\left[\bar{\Psi} H P_{R} \Psi+\bar{\Psi} H^{\dagger} P_{L} \Psi\right]-u \operatorname{tr}\left[H^{\dagger} H H^{\dagger} H\right]-v \operatorname{tr}\left[H^{\dagger} H\right] \operatorname{tr}\left[H^{\dagger} H\right]
\end{aligned}
$$

and exhibits a $\mathrm{U}\left(N_{c}\right) \times \mathrm{U}\left(N_{f}\right)_{L} \times \mathrm{U}\left(N_{f}\right)_{R}$ global symmetry. The Dirac fermions $\Psi$ and complex scalars $H$ are $N_{c} \times N_{f}$ and $N_{f} \times N_{f}$ matrix fields, respectively. $P_{L, R}=\frac{1}{2}\left(1 \mp \gamma_{5}\right)$ in (8.1) represents the left- and right chiral projectors. Moreover, we employ the limit $N_{f, c} \rightarrow \infty$, while the quantity

$$
\epsilon=\frac{N_{f}}{N_{c}}-\frac{11}{2}
$$

remains finite, along with the rescaled couplings

$$
\alpha_{y}=\frac{N_{c} y^{2}}{(4 \pi)^{2}}, \quad \alpha_{u}=\frac{N_{f} u}{(4 \pi)^{2}}, \quad \alpha_{v}=\frac{N_{f}^{2} v}{(4 \pi)^{2}} .
$$

In this limit, two-loop results are available from [51]. At three-loop, all unknown coefficients drop out and one obtains

$$
\begin{aligned}
\left.\gamma_{H}\right|_{3 \text {-loop }}= & -4 \alpha_{u}^{3}-\frac{15}{2} \alpha_{u}^{2} \alpha_{y}+\left[5 \alpha_{u}+\frac{1}{32}(183+10 \epsilon) \alpha_{y}\right]\left(\frac{11}{2}+\epsilon\right) \alpha_{y}^{2}, \\
\left.\gamma_{\Psi}\right|_{3 \text {-loop }}= & {\left[-\frac{11}{4} \alpha_{u}^{2}+(11+2 \epsilon) \alpha_{u} \alpha_{y}+\left(\frac{1217}{128}+\frac{41}{32} \epsilon-\frac{3}{32} \epsilon^{2}\right) \alpha_{y}^{2}\right]\left(\frac{11}{2}+\epsilon\right) \alpha_{y}, } \\
\left.\beta_{\alpha_{y}}\right|_{3 \text {-loop }}= & -8 \alpha_{u}^{3} \alpha_{y}+5\left(\frac{5}{2}+\epsilon\right) \alpha_{u}^{2} \alpha_{y}^{2}+12\left(\frac{11}{2}+\epsilon\right)(8+\epsilon) \alpha_{u} \alpha_{y}^{3} \\
& +\left(\frac{11}{2}+\epsilon\right)\left(\frac{1583}{32}+\frac{23}{4} \epsilon-\frac{3}{8} \epsilon^{2}\right) \alpha_{y}^{4}, \\
\left.\beta_{\alpha_{u}}\right|_{3 \text {-loop }}= & 104 \alpha_{u}^{4}+34 \alpha_{u}^{3} \alpha_{y}+(889+166 \epsilon) \alpha_{u}^{2} \alpha_{y}^{2}-\frac{1}{8}\left(\frac{11}{2}+\epsilon\right)^{2}(21-26 \epsilon) \alpha_{y}^{4} \\
& -\left(\frac{2601}{16}+\frac{283}{8} \epsilon+3 \zeta_{3}(11+2 \epsilon)\right)(11+2 \epsilon) \alpha_{u} \alpha_{y}^{3} \\
\left.\beta_{\alpha_{v}}\right|_{3 \text {-loop }}= & 12 \alpha_{v}^{2} \alpha_{u}^{2}+480 \alpha_{v} \alpha_{u}^{3}+\left(772+384 \zeta_{3}\right) \alpha_{u}^{4}+66 \alpha_{v} \alpha_{u}^{2} \alpha_{y}+192 \alpha_{u}^{3} \alpha_{y} \\
& +\left(\frac{427}{2}+41 \epsilon\right) \alpha_{v}^{2} \alpha_{y}^{2}+\left(788+152 \epsilon+96 \zeta_{3}\left(\frac{11}{2}+\epsilon\right)\right) \alpha_{v} \alpha_{u} \alpha_{y}^{2} \\
& +\left(\frac{1985}{2}+187 \epsilon+192 \zeta_{3}\left(\frac{11}{2}+\epsilon\right)\right) \alpha_{u}^{2} \alpha_{y}^{2} \\
& -\left(\frac{11}{2}+\epsilon\right)\left(552+112 \epsilon+60 \zeta_{3}\left(\frac{11}{2}+\epsilon\right)\right) \alpha_{u} \alpha_{y}^{3} \\
& -\frac{1}{8}\left(\frac{11}{2}+\epsilon\right)\left(1897+438 \epsilon-96 \zeta_{3}\left(\frac{11}{2}+\epsilon\right)\right) \alpha_{v} \alpha_{y}^{3} \\
& -3\left(\frac{11}{2}+\epsilon\right)\left(17+2 \epsilon+2 \zeta_{3}\left(\frac{11}{2}+\epsilon\right)\right) \alpha_{y}^{4},
\end{aligned}
$$




$$
\begin{aligned}
\left.m^{-2} \beta_{m^{2}}\right|_{3 \text {-loop }}= & 12 \alpha_{v} \alpha_{u}^{2}+240 \alpha_{u}^{3}+33 \alpha_{u}^{2} \alpha_{y}+\left(394+76 \epsilon+48 \zeta_{3}\left(\frac{11}{2}+\epsilon\right)\right) \alpha_{u} \alpha_{y}^{2} \\
& +\left(\frac{427}{2}+41 \epsilon\right) \alpha_{v} \alpha_{y}^{2}-\frac{1}{16}\left(\frac{11}{2}+\epsilon\right)\left(1897+438 \epsilon-96 \zeta_{3}\left(\frac{11}{2}+\epsilon\right)\right) \alpha_{y}^{3}
\end{aligned}
$$

as a consequence of $(4.2),(5.2),(6.2)$ and (7.2)ff. These results are compatible with [23, 26].

\section{Discussion and outlook}

In this work, we have investigated the three-loop renormalisation group evolution of general QFTs with scalars and fermions, featuring Yukawa, scalar quartic and cubic interactions, as well as fermion and scalar mass terms. Using literature results, we have completely determined the general result for all field anomalous dimensions, Yukawa and fermion mass $\beta$-functions, as well as terms $\propto \lambda^{4}, \propto y^{2} \lambda^{3}$ and $\propto y^{4} \lambda^{2}$ for scalar quartics. In the scalar potential, the number of unknown parameters has been vastly reduced to 13 , which can be fixed by future calculations. That is, either by direct computation of the diagrams listed herein, or as a side product of determining RGEs in specific theories. Unfortunately, there is little hope to utilise Weyl consistency conditions [12, 20, 21] to constrain these coefficients, as five- and four-loop gauge and Yukawa $\beta$-functions would be required. Rather, it is the other way around that computations of the latter profit from three-loop quartic RGEs. The results obtained here may already be sufficient to completely determine three-loop RGEs for certain QFTs. Trivial examples include the works [28-34] used as input, and it was demonstrated in section 8 that this selection is not exhaustive. In the absence of fermion masses, only three unknown parameters remain in the general $\mathrm{RG}$ evolution of scalar mass and cubic interactions.

Once the remaining parameters in this setup are determined, the next step towards a complete three-loop result would be the inclusion of gauge interactions. The gauge $\beta$ function itself is already known at three-loop [9-12], and progress towards a general fourloop expression is being made $[12,19,24,44,45]$. In particular, a basis of tensor structures has been formulated in [12], which also covers three-loop fermion and scalar anomalous dimensions, as well as Yukawa $\beta$-functions. The work spells out explicitly how involved the determination of these fully general RGEs is - an effort likely to be eclipsed by the threeloop quartic $\beta$-functions, for which a complete basis remains to be determined. This vision profits from the gaugeless contribution to be handled separately here in this work. As for the coefficients involving gauge interactions in $\gamma^{\phi, 3 \ell}, \gamma^{\psi, 3 \ell}$ and $\beta_{y^{a}}^{3 \ell}$, substantial amount may be fixed using the explicit computation [27] and the conditions in [12]. Likewise, as the SM gauge group is relatively large and structurally diverse, one should be cautiously optimistic that available results [28-32] determine a sizeable number of contributions to $\beta_{\lambda_{a b c d}}^{3 \ell}$.

\section{Acknowledgments}

The author is thankful to Ian Jack for comments on the manuscript as well as various insightful discussions, Dominik Stöckinger and Sandra Kvedaraitė for useful comments as well as Gustavo Medina Vazquez for proof-reading. 


\section{A Conversion to Weyl consistency condition basis}

In this appendix, we provide the results (6.2) in the basis of [12], wherein Weyl consistency condition between four-loop gauge, three-loop Yukawa and two-loop quartic RGEs have been studied. With the help of the program [16], we obtain the 86 coefficients

$$
\begin{aligned}
& \mathfrak{y}_{43}^{(3)}=-\frac{1}{16}, \quad \mathfrak{y}_{128}^{(3)}=\frac{3}{2}, \quad \mathfrak{y}_{129}^{(3)}=-\frac{3}{8}, \quad \mathfrak{y}_{130}^{(3)}=-\frac{11}{48}, \\
& \mathfrak{y}_{131}^{(3)}=1, \quad \mathfrak{y}_{132}^{(3)}=-\frac{5}{32}, \quad \mathfrak{y}_{224}^{(3)}=3, \quad \mathfrak{y}_{225}^{(3)}=-2, \\
& \mathfrak{y}_{226}^{(3)}=4, \quad \mathfrak{y}_{227}^{(3)}=5, \quad \mathfrak{y}_{228}^{(3)}=2, \quad \mathfrak{y}_{229}^{(3)}=6, \\
& \mathfrak{y}_{230}^{(3)}=2, \quad \mathfrak{y}_{231}^{(3)}=\frac{5}{8}, \quad \mathfrak{y}_{232}^{(3)}=\frac{5}{8}, \quad \mathfrak{y}_{233}^{(3)}=1, \\
& \mathfrak{y}_{234}^{(3)}=\frac{3}{2}, \quad \mathfrak{y}_{235}^{(3)}=1, \quad \mathfrak{y}_{236}^{(3)}=-3, \quad \mathfrak{y}_{237}^{(3)}=4\left(3 \zeta_{3}-1\right), \\
& \mathfrak{y}_{238}^{(3)}=6\left(2 \zeta_{3}-1\right), \quad \mathfrak{y}_{239}^{(3)}=4\left(3 \zeta_{3}-2\right), \quad \mathfrak{y}_{240}^{(3)}=2, \quad \mathfrak{y}_{241}^{(3)}=2, \\
& \mathfrak{y}_{242}^{(3)}=4\left(3 \zeta_{3}-1\right), \quad \mathfrak{y}_{243}^{(3)}=6\left(2 \zeta_{3}-1\right), \quad \mathfrak{y}_{244}^{(3)}=6 \zeta_{3}-5, \quad \mathfrak{y}_{245}^{(3)}=3 \zeta_{3}-2, \\
& \mathfrak{y}_{246}^{(3)}=\frac{3}{2} \zeta_{3}-1, \quad \mathfrak{y}_{247}^{(3)}=-3, \quad \mathfrak{y}_{248}^{(3)}=-1, \quad \mathfrak{y}_{249}^{(3)}=-1, \\
& \mathfrak{y}_{250}^{(3)}=-8, \quad \mathfrak{y}_{251}^{(3)}=-6, \quad \mathfrak{y}_{252}^{(3)}=0, \quad \mathfrak{y}_{253}^{(3)}=-2, \\
& \mathfrak{y}_{254}^{(3)}=-4, \quad \mathfrak{y}_{255}^{(3)}=2, \quad \mathfrak{y}_{256}^{(3)}=4, \quad \mathfrak{y}_{257}^{(3)}=\frac{5}{4}, \\
& \mathfrak{y}_{258}^{(3)}=-2, \quad \mathfrak{y}_{259}^{(3)}=4, \quad \mathfrak{y}_{260}^{(3)}=-2, \quad \mathfrak{y}_{261}^{(3)}=-\frac{5}{8}, \\
& \mathfrak{y}_{262}^{(3)}=1, \quad \mathfrak{y}_{263}^{(3)}=1, \quad \mathfrak{y}_{264}^{(3)}=0, \quad \mathfrak{y}_{265}^{(3)}=0, \\
& \mathfrak{y}_{266}^{(3)}=-\frac{3}{8}, \quad \mathfrak{y}_{267}^{(3)}=-\frac{3}{4}, \quad \mathfrak{y}_{268}^{(3)}=\frac{7}{4}, \quad \mathfrak{y}_{269}^{(3)}=1, \\
& \mathfrak{y}_{270}^{(3)}=\frac{25}{8}, \quad \mathfrak{y}_{271}^{(3)}=\frac{7}{8}, \quad \mathfrak{y}_{272}^{(3)}=-3, \quad \mathfrak{y}_{273}^{(3)}=4 \\
& \mathfrak{y}_{274}^{(3)}=3, \quad \mathfrak{y}_{275}^{(3)}=-2, \quad \mathfrak{y}_{276}^{(3)}=-3, \quad \mathfrak{y}_{277}^{(3)}=\frac{3}{16}, \\
& \mathfrak{y}_{278}^{(3)}=\frac{1}{2}, \quad \mathfrak{y}_{279}^{(3)}=2, \quad \mathfrak{y}_{280}^{(3)}=\frac{1}{8}, \quad \mathfrak{y}_{281}^{(3)}=\frac{3}{16}, \\
& \mathfrak{y}_{282}^{(3)}=\frac{7}{16}, \quad \mathfrak{y}_{283}^{(3)}=\frac{5}{16}, \quad \mathfrak{y}_{284}^{(3)}=\frac{7}{16}, \quad \mathfrak{y}_{285}^{(3)}=2, \\
& \mathfrak{y}_{286}^{(3)}=\frac{25}{8}, \quad \mathfrak{y}_{287}^{(3)}=-3, \quad \mathfrak{y}_{288}^{(3)}=3, \quad \mathfrak{y}_{289}^{(3)}=-1, \\
& \mathfrak{y}_{290}^{(3)}=-\frac{3}{16}, \quad \mathfrak{y}_{291}^{(3)}=-\frac{9}{16}, \quad \mathfrak{y}_{292}^{(3)}=-\frac{3}{16}, \quad \mathfrak{y}_{293}^{(3)}=\frac{9}{16}, \\
& \mathfrak{y}_{294}^{(3)}=1, \quad \mathfrak{y}_{295}^{(3)}=-\frac{1}{2}, \quad \mathfrak{y}_{296}^{(3)}=-1, \quad \mathfrak{y}_{297}^{(3)}=-\frac{5}{16}, \\
& \mathfrak{y}_{298}^{(3)}=\frac{1}{32}, \quad \mathfrak{y}_{299}^{(3)}=-\frac{3}{16}, \quad \mathfrak{y}_{300}^{(3)}=-1, \quad \mathfrak{y}_{301}^{(3)}=-\frac{1}{4}, \\
& \mathfrak{y}_{302}^{(3)}=-\frac{1}{2}, \quad \mathfrak{y}_{303}^{(3)}=-\frac{3}{16} .
\end{aligned}
$$

Our findings are not in contradiction to the general 265 Weyl consistency condition formulated in [12]. In fact, $\mathfrak{y}_{226}^{(3)}$ and $\mathfrak{y}_{228}^{(3)}$ fit their direct prediction therein. While the two-loop 
quartic RGEs merely reduce the number of conditions by 4, the results (A.1) leave only 219 such conditions. In particular, the direct predictions of coefficients are updated to

$$
\begin{array}{rlrlrl}
\mathfrak{g}_{55}^{(4)}=-\frac{27}{2}, & \mathfrak{g}_{60}^{(4)}=\frac{1}{2}, & \mathfrak{g}_{67}^{(4)}=-\frac{1}{12}, & \mathfrak{g}_{110}^{(4)}=-4, & \mathfrak{g}_{112}^{(4)}=\frac{1}{4}, \\
\mathfrak{g}_{158}^{(4)}=0, & \mathfrak{g}_{160}^{(4)}=-\frac{5}{2}, & \mathfrak{y}_{2}^{(3)}=-3, & \mathfrak{y}_{4}^{(3)}=-\frac{3}{2}, & \mathfrak{y}_{39}^{(3)}=-12, \\
\mathfrak{y}_{40}^{(3)}=\frac{5}{4}, & \mathfrak{y}_{41}^{(3)}=-\frac{17}{48}, & \mathfrak{y}_{42}^{(3)}=\frac{19}{16}, & \mathfrak{y}_{47}^{(3)}=\frac{3}{4}, & \mathfrak{y}_{49}^{(3)}=0, \\
\mathfrak{y}_{122}^{(3)}=0, & \mathfrak{y}_{123}^{(3)}=-\frac{17}{2}, & \mathfrak{y}_{124}^{(3)}=-17, & \mathfrak{y}_{125}^{(3)}=\frac{19}{2}, & \mathfrak{y}_{126}^{(3)}=2, \\
\mathfrak{y}_{127}^{(3)}=0 . & & &
\end{array}
$$

\section{B Comparison to supersymmetric RGEs}

The non-renormalisation theorem $[52,53]$ suggests that only superfield anomalous dimensions $\gamma_{B}^{A}$, contribute to the $\beta$-functions of superpotential parameters, while quantum corrections to the vertex renormalisations are finite. Expressions for the superfield anomalous dimensions are available at three-loop $[35,36]$ or using dimensional reduction (DRED) [54, $55]$ and the $\overline{\mathrm{DR}}$ scheme. In renormalisable QFTs without gauge interactions, DRED is equivalent to DREG since no $\epsilon$-scalars are introduced, up to ambiguities due to the $\gamma_{5}$ which are absent in our setup as argued earlier. ${ }^{2}$ At two-loop order, this is verified explicitly by the scheme conversions provided by [57]. Therefore, we can express $\mathcal{N}=1$ QFTs in the language of a non-supersymmetric ones, see for instance [57, 58], and directly compare our $\overline{\mathrm{MS}}$ ansatz against the supersymmetric RGEs in the $\overline{\mathrm{DR}}$ scheme - similar to the conduct in $[5$, 18, 21]. The perturbatively renormalisable $\mathcal{N}=1$ superpotential of dimensionless couplings

$$
W=\frac{1}{6} Y^{A B C} \Phi_{A} \Phi_{B} \Phi_{C},
$$

only contains Yukawa interactions $Y^{A B C}$ and is holomorphic in the chiral superfields $\Phi_{A}$. Here, the corresponding complex conjugate Yukawa interaction will be denoted as $Y_{A B C}$, counting only over antichiral fields $\Phi^{A}$. Each superfield $\Phi_{A}$ contains the complex scalar $\phi_{A}$ and Weyl fermion $\psi_{A}$ as well as auxiliary fields. Integrating the latter out from the action, one obtains the Yukawa and quartic interactions

$$
\frac{1}{2} Y^{A B C}\left(\phi_{A} \psi_{B}^{\top} \varepsilon \psi_{C}\right)+\text { h.c. } \quad \text { and } \quad \frac{1}{4} Y^{A B E} Y_{E C D}\left(\phi_{A} \phi_{B} \phi_{C}^{*} \phi_{D}^{*}\right) .
$$

As the $\beta$-function for the Yukawa interaction is given by

$$
\beta^{A B C}=\gamma^{A}{ }_{D} Y^{D B C}+\gamma^{B}{ }_{D} Y^{A D C}+\gamma^{C}{ }_{D} Y^{A B D},
$$

the renormalisation group evolution of the quartic $\lambda^{A B}{ }_{C D}=Y^{A B E} Y_{E C D}$ is constructed via

$$
\begin{aligned}
\beta_{C D}^{A B}= & \left(\gamma_{F}^{A} Y^{F B E}+\gamma_{F}^{B} Y^{A F E}\right) Y_{E C D}+Y^{A B E}\left(Y_{E F D} \gamma_{C}^{F}+Y_{E C F} \gamma^{F}{ }_{D}\right) \\
& +2 Y^{A B F} \gamma_{F}^{E} Y_{E C D} .
\end{aligned}
$$

\footnotetext{
${ }^{2}$ See [56] for a recent overview of various regularisation schemes.
} 


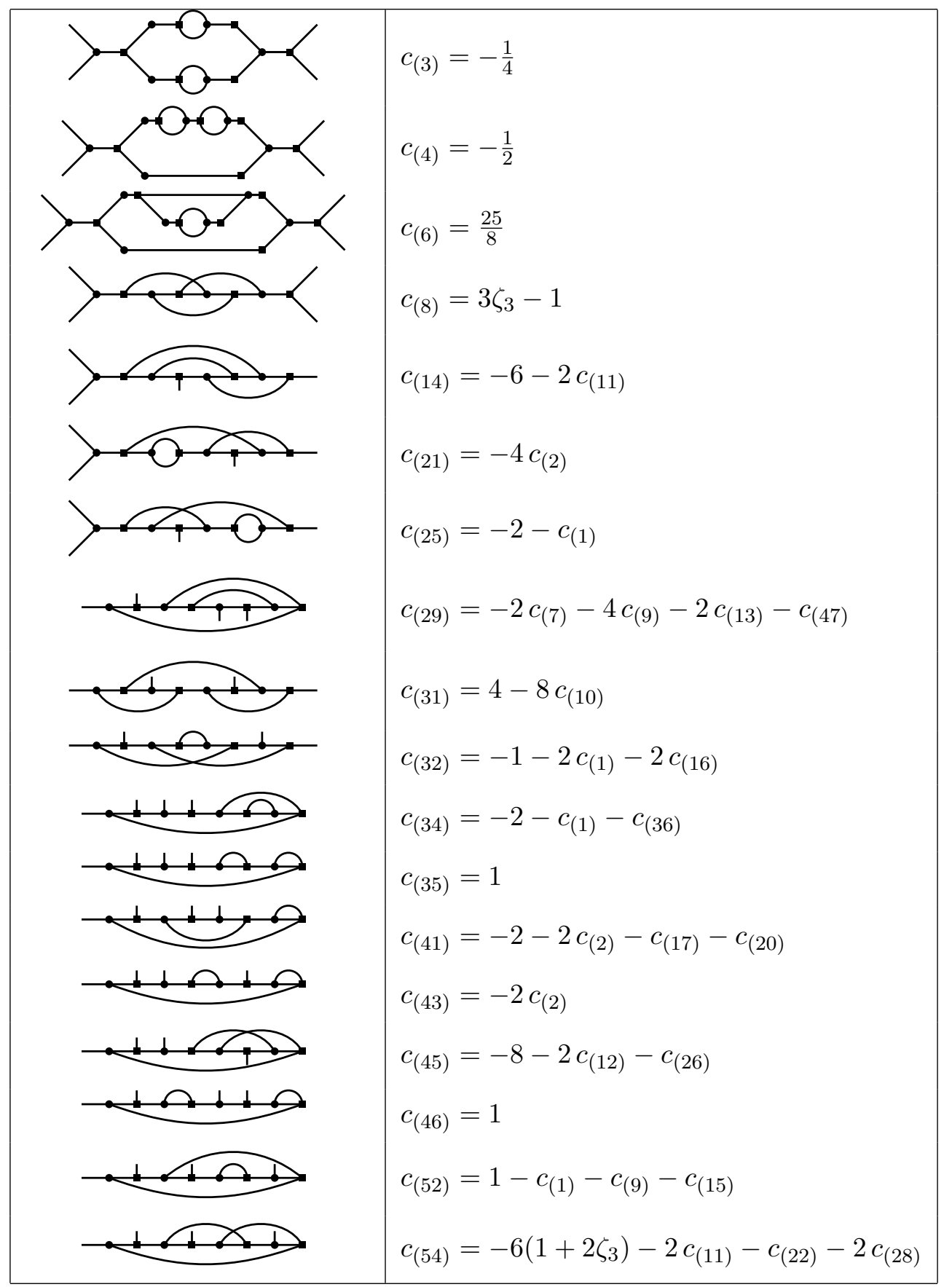

Table 1. Relations between coefficients for tensor structures in figure 4 and figure 5 (left), inferred from comparison with supersymmetric RGEs. The corresponding graphs on the right contain round vertices denoting an insertion of $Y^{A B C}$ while squared nodes mark $Y_{A B C}$ interactions. 
Here, $\gamma_{B}^{A}$ denotes the chiral superfield anomalous dimensions, with the three-loop part given by $[35,36]$

$$
\begin{aligned}
(4 \pi)^{6} \gamma_{B}^{A}= & Y^{A C D} Y_{D E F} Y^{F G H} Y_{G H I} Y^{E I J} Y_{B C J} \\
& -\frac{1}{8} Y^{A C D} Y_{C E F} Y^{E F G} Y_{D H I} Y^{H I J} Y_{B G J} \\
& -\frac{1}{4} Y^{A C D} Y_{D E F} Y^{E F G} Y_{G H I} Y^{H I J} Y_{B C J} \\
& +\frac{3}{2} \zeta_{3} Y^{A C D} Y_{C E F} Y_{D G H} Y^{E G I} Y^{F H J} Y_{B I J}
\end{aligned}
$$

To compare to non-supersymmetric RGEs, the QFT defined by the action

$$
\begin{aligned}
\mathcal{L}= & \partial^{\mu} \phi_{A}^{*} \partial_{\mu} \phi_{A}+i \psi_{A}^{\dagger} \sigma^{\mu} \partial_{\mu} \psi_{A} \\
& -\frac{1}{2}\left[y^{A B C}\left(\phi_{A} \psi_{B}^{\top} \varepsilon \psi_{C}\right)+y^{* A B C}\left(\phi_{A}^{*} \psi_{B}^{\dagger} \varepsilon \psi_{C}^{*}\right)\right]-\frac{1}{4} \lambda^{A B}{ }_{C D}\left(\phi_{A} \phi_{B} \phi_{C}^{*} \phi_{D}^{*}\right),
\end{aligned}
$$

consisting of Weyl fermions $\psi_{A}$ and complex scalars $\phi_{A}$ is analysed. The $\beta$-function of the quartic $\lambda_{C D}^{A B}$ can be computed using eq. (7.2)ff. After insertion of the supersymmetry relations

$$
y^{A B C}=Y^{A B C}, \quad y^{* A B C}=Y_{A B C}, \quad \lambda^{A B}{ }_{C D}=Y^{A B E} Y_{E C D},
$$

the result can be directly compared to (B.4), constraining unknown coefficients $c_{(n)}$ of tensor structures $(n)$ in figure 4 and figure 5 . However, certain features are inaccessible: contractions (5), (18), (19), (23), (24), (27), (30), (33), (37)-(40), (42), (44), (48)-(51), (53), (55) and (56) vanish, since all scalars are complex and the Yukawa interaction reflects the holomorphism of the superpotential. Choosing a basis of contractions of $Y^{A B C}$ and $Y_{A B C}$, after the insertion of (B.7), the extracted relations are listed in table 1.

Open Access. This article is distributed under the terms of the Creative Commons Attribution License (CC-BY 4.0), which permits any use, distribution and reproduction in any medium, provided the original author(s) and source are credited.

\section{References}

[1] M.E. Machacek and M.T. Vaughn, Two loop renormalization group equations in a general quantum field theory. 1. Wave function renormalization, Nucl. Phys. B 222 (1983) 83 [INSPIRE].

[2] M.E. Machacek and M.T. Vaughn, Two loop renormalization group equations in a general quantum field theory. 2. Yukawa couplings, Nucl. Phys. B 236 (1984) 221 [InSPIRE].

[3] M.E. Machacek and M.T. Vaughn, Two loop renormalization group equations in a general quantum field theory. 3. Scalar quartic couplings, Nucl. Phys. B 249 (1985) 70 [inSPIRE].

[4] M.-X. Luo, H.-W. Wang and Y. Xiao, Two loop renormalization group equations in general gauge field theories, Phys. Rev. D 67 (2003) 065019 [hep-ph/0211440] [INSPIRE].

[5] I. Schienbein, F. Staub, T. Steudtner and K. Svirina, Revisiting RGEs for general gauge theories, Nucl. Phys. B 939 (2019) 1 [Erratum ibid. 966 (2021) 115339] [arXiv:1809.06797] [INSPIRE]. 
[6] M. Sperling, D. Stöckinger and A. Voigt, Renormalization of vacuum expectation values in spontaneously broken gauge theories, JHEP 07 (2013) 132 [arXiv:1305.1548] [INSPIRE].

[7] M. Sperling, D. Stöckinger and A. Voigt, Renormalization of vacuum expectation values in spontaneously broken gauge theories: two-loop results, JHEP 01 (2014) 068 [arXiv:1310.7629] [INSPIRE].

[8] L. Sartore, General renormalization group equations for dimensionful couplings in the $\overline{\mathrm{MS}}$ scheme, Phys. Rev. D 102 (2020) 076002 [arXiv:2006.12307] [InSPIRE].

[9] A.G.M. Pickering, J.A. Gracey and D.R.T. Jones, Three loop gauge $\beta$-function for the most general single gauge coupling theory, Phys. Lett. B 510 (2001) 347 [Erratum ibid. 535 (2002) 377] [hep-ph/0104247] [INSPIRE].

[10] L.N. Mihaila, J. Salomon and M. Steinhauser, Renormalization constants and $\beta$-functions for the gauge couplings of the Standard Model to three-loop order, Phys. Rev. D 86 (2012) 096008 [arXiv: 1208.3357] [INSPIRE].

[11] L. Mihaila, Three-loop gauge $\beta$-function in non-simple gauge groups, PoS (RADCOR2013) 060 (2013) [INSPIRE].

[12] C. Poole and A.E. Thomsen, Constraints on 3- and 4-loop $\beta$-functions in a general four-dimensional quantum field theory, JHEP 09 (2019) 055 [arXiv: 1906.04625] [INSPIRE].

[13] F. Staub, SARAH 4: a tool for (not only SUSY) model builders, Comput. Phys. Commun. 185 (2014) 1773 [arXiv: 1309.7223] [INSPIRE].

[14] L. Sartore and I. Schienbein, PyR@TE 3, Comput. Phys. Commun. 261 (2021) 107819 [arXiv:2007.12700] [INSPIRE].

[15] D.F. Litim and T. Steudtner, ARGES - Advanced Renormalisation Group Equation Simplifier, arXiv:2012.12955 [INSPIRE].

[16] A.E. Thomsen, RGBeta: a Mathematica package for the evaluation of renormalization group $\beta$-functions, arXiv:2101.08265 [INSPIRE].

[17] F. Jegerlehner, Facts of life with $\gamma_{5}$, Eur. Phys. J. C 18 (2001) 673 [hep-th/0005255] [INSPIRE].

[18] I. Jack and C. Poole, The a-function for gauge theories, JHEP 01 (2015) 138 [arXiv:1411.1301] [INSPIRE].

[19] C. Poole and A.E. Thomsen, Weyl consistency conditions and $\gamma_{5}$, Phys. Rev. Lett. 123 (2019) 041602 [arXiv: 1901.02749] [INSPIRE].

[20] H. Osborn, Weyl consistency conditions and a local renormalization group equation for general renormalizable field theories, Nucl. Phys. B 363 (1991) 486 [InSPIRE].

[21] I. Jack and H. Osborn, Constraints on $R G$ flow for four dimensional quantum field theories, Nucl. Phys. B $\mathbf{8 8 3}$ (2014) 425 [arXiv:1312.0428] [INSPIRE].

[22] I. Jack and C. Poole, Scheme invariants in $\phi^{4}$ theory in four dimensions, Phys. Rev. D 98 (2018) 065011 [arXiv:1806.08598] [INSPIRE].

[23] T. Steudtner, General scalar renormalisation group equations at three-loop order, JHEP 12 (2020) 012 [arXiv: 2007.06591] [INSPIRE].

[24] J. Davies, F. Herren, C. Poole, M. Steinhauser and A.E. Thomsen, Gauge coupling $\beta$ functions to four-loop order in the Standard Model, Phys. Rev. Lett. 124 (2020) 071803 [arXiv: 1912.07624] [INSPIRE]. 
[25] I. Jack and H. Osborn, Analogs for the $c$ theorem for four-dimensional renormalizable field theories, Nucl. Phys. B 343 (1990) 647 [INSPIRE].

[26] A. Bednyakov and A. Pikelner, Six-loop $\beta$-functions in general scalar theory, JHEP 04 (2021) 233 [arXiv :2102.12832] [INSPIRE].

[27] F. Herren, L. Mihaila and M. Steinhauser, Gauge and Yukawa coupling $\beta$-functions of two-Higgs-doublet models to three-loop order, Phys. Rev. D 97 (2018) 015016 [Erratum ibid. 101 (2020) 079903] [arXiv:1712.06614] [INSPIRE].

[28] K.G. Chetyrkin and M.F. Zoller, Three-loop $\beta$-functions for top-Yukawa and the Higgs self-interaction in the Standard Model, JHEP 06 (2012) 033 [arXiv:1205.2892] [INSPIRE].

[29] A.V. Bednyakov, A.F. Pikelner and V.N. Velizhanin, Higgs self-coupling $\beta$-function in the Standard Model at three loops, Nucl. Phys. B 875 (2013) 552 [arXiv:1303.4364] [INSPIRE].

[30] K.G. Chetyrkin and M.F. Zoller, $\beta$-function for the Higgs self-interaction in the Standard Model at three-loop level, JHEP 04 (2013) 091 [Erratum ibid. 09 (2013) 155] [arXiv:1303.2890] [INSPIRE].

[31] A.V. Bednyakov, A.F. Pikelner and V.N. Velizhanin, Three-loop Higgs self-coupling $\beta$-function in the Standard Model with complex Yukawa matrices, Nucl. Phys. B 879 (2014) 256 [arXiv: 1310.3806] [INSPIRE].

[32] A.V. Bednyakov, A.F. Pikelner and V.N. Velizhanin, Three-loop SM $\beta$-functions for matrix Yukawa couplings, Phys. Lett. B 737 (2014) 129 [arXiv:1406.7171] [INSPIRE].

[33] N. Zerf, L.N. Mihaila, P. Marquard, I.F. Herbut and M.M. Scherer, Four-loop critical exponents for the Gross-Neveu-Yukawa models, Phys. Rev. D 96 (2017) 096010 [arXiv: 1709.05057] [INSPIRE].

[34] L.N. Mihaila, N. Zerf, B. Ihrig, I.F. Herbut and M.M. Scherer, Gross-Neveu-Yukawa model at three loops and Ising critical behavior of Dirac systems, Phys. Rev. B 96 (2017) 165133 [arXiv: 1703.08801] [INSPIRE].

[35] I. Jack, D.R.T. Jones and C.G. North, $N=1$ supersymmetry and the three loop anomalous dimension for the chiral superfield, Nucl. Phys. B 473 (1996) 308 [hep-ph/9603386] [INSPIRE].

[36] A.J. Parkes, Three loop finiteness conditions in $N=1$ super Yang-Mills, Phys. Lett. B 156 (1985) 73 [INSPIRE].

[37] C.G. Bollini and J.J. Giambiagi, Lowest order "divergent" graphs in $\nu$-dimensional space, Phys. Lett. B 40 (1972) 566 [InSPIRE].

[38] C.G. Bollini and J.J. Giambiagi, Dimensional renormalization: the number of dimensions as a regularizing parameter, Nuovo Cim. B 12 (1972) 20 [INSPIRE].

[39] G. 't Hooft, Dimensional regularization and the renormalization group, Nucl. Phys. B 61 (1973) 455 [INSPIRE].

[40] W.A. Bardeen, A.J. Buras, D.W. Duke and T. Muta, Deep inelastic scattering beyond the leading order in asymptotically free gauge theories, Phys. Rev. D 18 (1978) 3998 [InSPIRE].

[41] S.P. Martin and M.T. Vaughn, Two loop renormalization group equations for soft supersymmetry breaking couplings, Phys. Rev. D 50 (1994) 2282 [Erratum ibid. 78 (2008) 039903] [hep-ph/9311340] [INSPIRE]. 
[42] G. 't Hooft and M.J.G. Veltman, Regularization and renormalization of gauge fields, Nucl. Phys. B 44 (1972) 189 [inSPIRE].

[43] H. Bélusca-Maïto, A. Ilakovac, M. Mađor-Božinović and D. Stöckinger, Dimensional regularization and Breitenlohner-Maison/'t Hooft-Veltman scheme for $\gamma_{5}$ applied to chiral YM theories: full one-loop counterterm and RGE structure, JHEP 08 (2020) 024 [arXiv:2004.14398] [INSPIRE].

[44] A.V. Bednyakov and A.F. Pikelner, Four-loop strong coupling $\beta$-function in the Standard Model, Phys. Lett. B 762 (2016) 151 [arXiv:1508.02680] [InSPIRE].

[45] M.F. Zoller, Top-Yukawa effects on the $\beta$-function of the strong coupling in the SM at four-loop level, JHEP 02 (2016) 095 [arXiv: 1508.03624] [INSPIRE].

[46] A.V. Bednyakov, A.F. Pikelner and V.N. Velizhanin, Yukawa coupling $\beta$-functions in the Standard Model at three loops, Phys. Lett. B 722 (2013) 336 [arXiv:1212.6829] [INSPIRE].

[47] I. Jack and H. Osborn, Scheme dependence and multiple couplings, arXiv:1606.02571 [INSPIRE].

[48] A.V. Bednyakov, On three-loop RGE for the Higgs sector of 2HDM, JHEP 11 (2018) 154 [arXiv: 1809.04527] [INSPIRE].

[49] D.F. Litim and F. Sannino, Asymptotic safety guaranteed, JHEP 12 (2014) 178 [arXiv: 1406.2337] [INSPIRE].

[50] D.F. Litim, M. Mojaza and F. Sannino, Vacuum stability of asymptotically safe gauge-Yukawa theories, JHEP 01 (2016) 081 [arXiv:1501.03061] [INSPIRE].

[51] A.D. Bond, D.F. Litim, G. Medina Vazquez and T. Steudtner, UV conformal window for asymptotic safety, Phys. Rev. D 97 (2018) 036019 [arXiv:1710.07615] [InSPIRE].

[52] A. Salam and J.A. Strathdee, On superfields and Fermi-Bose symmetry, Phys. Rev. D 11 (1975) 1521 [INSPIRE].

[53] M.T. Grisaru, W. Siegel and M. Roček, Improved methods for supergraphs, Nucl. Phys. B 159 (1979) 429 [INSPIRE].

[54] W. Siegel, Supersymmetric dimensional regularization via dimensional reduction, Phys. Lett. B 84 (1979) 193 [INSPIRE].

[55] D.M. Capper, D.R.T. Jones and P. van Nieuwenhuizen, Regularization by dimensional reduction of supersymmetric and nonsupersymmetric gauge theories, Nucl. Phys. B 167 (1980) 479 [INSPIRE].

[56] C. Gnendiger et al., To d, or not to d: recent developments and comparisons of regularization schemes, Eur. Phys. J. C $\mathbf{7 7}$ (2017) 471 [arXiv:1705.01827] [InSPIRE].

[57] S.P. Martin and M.T. Vaughn, Regularization dependence of running couplings in softly broken supersymmetry, Phys. Lett. B 318 (1993) 331 [hep-ph/9308222] [INSPIRE].

[58] S.P. Martin, A supersymmetry primer, Adv. Ser. Direct. High Energy Phys. 18 (1998) 1 [Adv. Ser. Direct. High Energy Phys. 21 (2010) 1] [hep-ph/9709356] [InSPIRE]. 